Document downloaded from:

http://hdl.handle.net/10251/80743

This paper must be cited as:

Ródenas García, JJ.; Bugeda Castelltort, G.; Albelda Vitoria, J.; Oñate Ibáñez De Navarra, E. (2011). On the need for the use of error-controlled finite element analyses in structural shape optimization processes. International Journal for Numerical Methods in Engineering. 87(11):1105-1126. doi:10.1002/nme.3155.

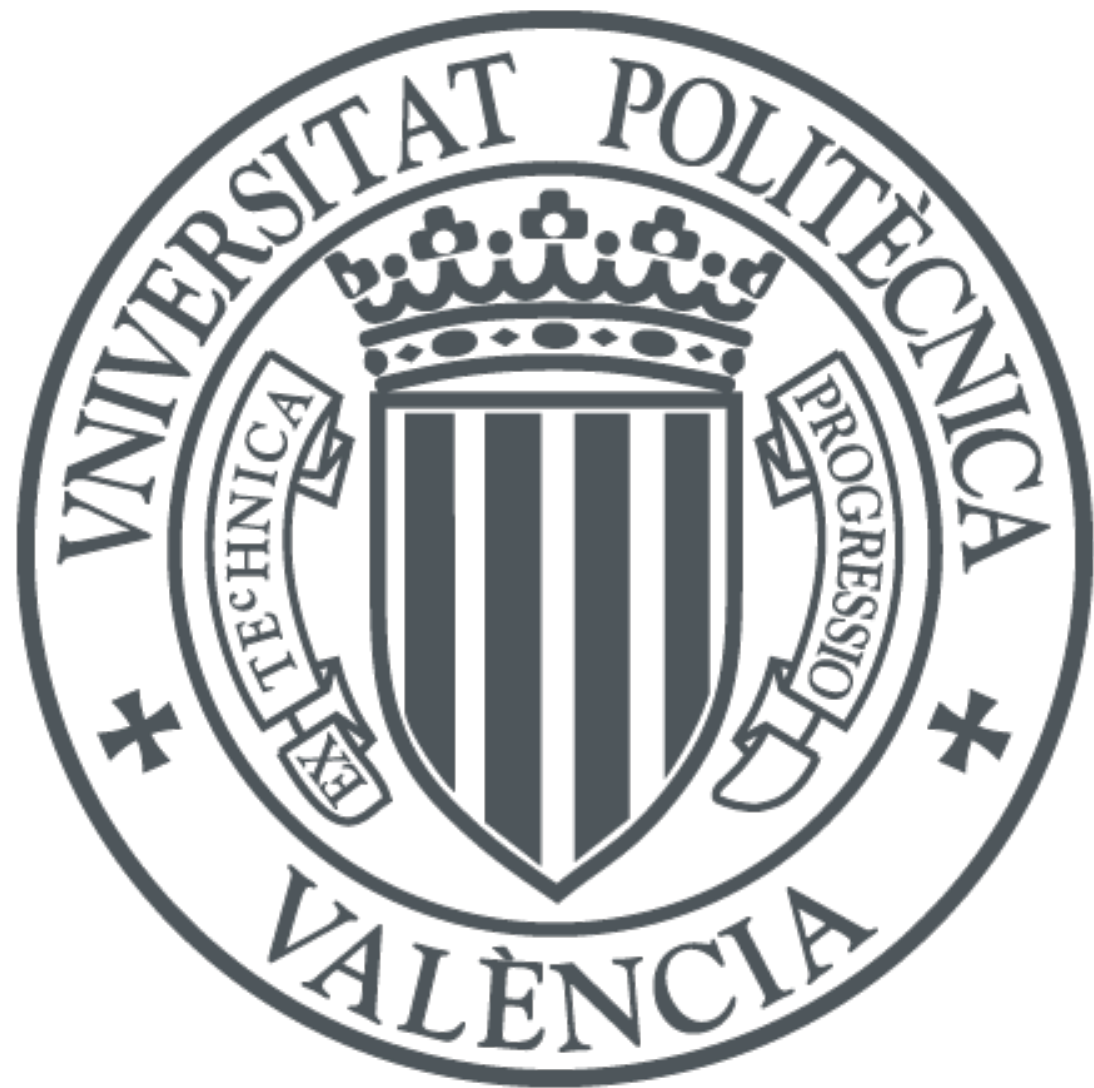

The final publication is available at

http://dx.doi.org/10.1002/nme.3155

Copyright Wiley

Additional Information

This is the peer reviewed version of the following article: Ródenas, J. J., Bugeda, G., Albelda, J. and Oñate, E. (2011), On the need for the use of error-controlled finite element analyses in structural shape optimization processes. Int. J. Numer. Meth. Engng., 87: 1105-1126, which has been published in final form at http://dx.doi.org/10.1002/nme.3155. This article may be used for non-commercial purposes in accordance with Wiley Terms and Conditions for Self-Archiving. 


\title{
On the need for the use of error-controlled finite element analyses in structural shape optimization processes
}

\author{
Short title: Error-controlled FE analyses in structural shape optimization \\ J.J. Ródenas ${ }^{1, * \dagger}$, G. Bugeda ${ }^{2, \ddagger}$, J. Albelda ${ }^{1, \S}$ and E. Oñate ${ }^{3, * *}$ \\ ${ }^{1}$ Centro de Investigación de Tecnología de Vehículos (CITV). \\ Universidad Politécnica de Valencia. Valencia - Spain \\ ${ }^{2}$ Escola Universitària d’Enginyeria Tècnica Industrial de Barcelona (EUETIB). \\ Universitat Politècnica de Catalunya. Barcelona - Spain \\ ${ }^{3}$ International Center for Numerical Methods in Engineering (CIMNE). \\ Universitat Politècnica de Catalunya. Barcelona - Spain
}

\begin{abstract}
SUMMARY
This work analyzes the influence of the discretization error associated to the Finite Element (FE) analyses of each design configuration proposed by the structural shape optimization algorithms over the behavior of the algorithm. The paper clearly shows that if FE analyses are not accurate enough, the final solution provided by the optimization algorithm will neither be optimal nor satisfy the constraints. The need for the use of adaptive FE analysis techniques in shape optimum design will be shown. The paper proposes the combination of two strategies to reduce the computational cost related to the use of mesh adaptivity in evolutionary optimization algorithms: (a) the use of an algorithm for the mesh generation by projection of the discretization error, which reduces the computational cost associated to the adaptive FE analysis of each geometrical configuration and, $(b)$ the successive increase of the required accuracy of the FE analyses in order to obtain a considerable reduction of the computational cost in the early stages of the optimization process.
\end{abstract}

KEY WORDS: Structural shape optimization; Error estimation; Adaptive remeshing; Sensitivity analysis; Evolutionary algorithms; Differential evolution

\section{INTRODUCTION}

An optimization problem can be mathematically viewed as the minimization of a function $f(\mathbf{v})$ depending on a set of variables $\mathbf{v}$ and subjected to some constraints. The general form of such a problem is:

\footnotetext{
* Correspondence to: Juan José Ródenas, Centro de Investigación de Tecnología de Vehículos. Departamento de Ingeniería Mecánica y de Materiales, Universidad Politécnica de Valencia. Camino de Vera s/n. 46022-Valencia (Spain). Tel +34 963877621 Fax +34 963877629

† e-mail: jjrodena@mcm.upv.es

\$ e-mail: gabriel.bugeda@upc.edu

$\S$ e-mail: jalbelda@mcm.upv.es

**e-mail: onate@cimne.upc.edu
} 


$$
\begin{array}{ll}
\text { minimize : } & f(\mathbf{v}) ; \mathbf{v}=\left\{v_{i}\right\} ; i=1, \ldots, n \\
\text { with : } & \mathbf{g}(\mathbf{v})=\left\{g_{j}(\mathbf{v})\right\} ; j=1, \ldots, m \\
\text { verifying : } & g_{j}(\mathbf{v}) \leq 0 \quad ; j=1, \ldots, m \\
& a_{i} \leq v_{i} \leq b_{i} \quad ; i=1, \ldots, n
\end{array}
$$

where $f$ is the objective function (OF), $v_{i}$ are the design variables and $g_{j}$ are inequality constraints which, for structural problems, are normally expressed in terms of stresses and/or displacements. The values $a_{i}$ and $b_{i}$ define lateral constraints. Each individual is characterized by a set of values of $\mathbf{v}$ that correspond to a specific structural design. The definition of each design in terms of the values of $\mathbf{v}$ is called the parameterization of the optimization problem. The resolution of the optimum design problem consists of finding the values of $\mathbf{v}$ defining the best design.

The algorithms used to solve optimization problems are generally iterative. Whichever the algorithm used, it is necessary to evaluate the values $f$ and $\mathbf{g}$ for each of the different designs during the iterative optimization process. In this work we have considered structural shape optimization problems. The values for $f$ and $\mathbf{g}$ in this kind of problems are usually obtained by the use of the finite element method (FEM). Hence, one should create a specific mesh for each of the different designs to be analyzed and then use the FEM to obtain the structural response of each design and, if necessary, the corresponding sensitivities with respect to the design variables. Two main aspects relative to the evaluation of $f$ and $\mathbf{g}$ by means of the FEM, which have a great importance over the global behavior of the optimization process, must be taken into account: the computational effort required for the numerical evaluation of each individual (geometrical configuration) and the accuracy of the FEM results.

The importance of the computational effort required for the evaluation of each geometrical configuration is evident. In optimization problems like those under consideration, most of the computational cost is devoted to the analysis of individuals in order to obtain the values of the OF, the degree of satisfaction of the constraints and, eventually, their corresponding sensitivity analysis.

On the other hand, and related to the accuracy of the results, it must be taken into account that these numerical analysis techniques provide only approximate values for the data required by the optimization algorithm. If these values are not accurate enough, an excessive amount of noise can be introduced in the optimization process. This could decrease the rate of convergence of the optimization process, and could cause the convergence to a solution that is non-optimal or unfeasible. In the context of the FEM, the so called $h$-adaptive techniques, the $p$-adaptive techniques and the $h p$-adaptive techniques can be used to obtain solutions with the prescribed accuracy level. However, the use of these techniques implies a big computational cost that reduces the computational efficiency of the optimization process.

This paper will show that the correct behavior of the optimization process is only ensured if a minimum quality of the results of the analysis of each design, used to drive the optimization process, is ensured. To do this, the effect of the prescribed maximum error in energy norm over the final results obtained by means of a gradient-based deterministic algorithm and those obtained with an evolutionary algorithm, will be shown.

As previously commented, adaptive FEM techniques can be used to obtain solutions with the prescribed accuracy level. However, the use of the traditional adaptive 
techniques implies the successive analysis of a sequence of numerical models with increasing accuracy, automatically adapted to the characteristics of the solution of the problem. The computational cost related to the use of these traditional adaptive analysis techniques can be critical when evolutionary algorithms are used. To solve this problem, in this paper we propose the use of the $h$-adaptive analysis technique for generations of individuals described in [1]. The procedure consists of using the sensitivity analysis of the discretization error in energy norm with respect to the design variables [2-4] to project the error obtained from a representative individual of the generation into the different geometrical configurations to be analyzed. $H$-adapted meshes for the analysis of each particular individual can be created with this information, which in the vast majority of cases provides FE results with the prescribed accuracy, thus avoiding the high computational cost associated to the full $h$-adaptive remeshing.

\section{CASE STUDY AND MOTIVATION. PIPE CROSS SECTION}

This section shows the results corresponding to the solution of an optimization problem, with a known analytical solution, using different levels of the controlled discretization error as a motivation for the developments presented in this paper. Both, evolutionary and deterministic optimization algorithms have been considered. The part to be optimized corresponds to the cross-section of a pipe subjected to an internal pressure. Two perpendicular planes of symmetry have been considered; therefore, only one quarter of the section has been modeled, as shown in Fig. 1. The shape is defined by a total of 8 points, 5 of these points describe a circular internal shape and 3 describe the external boundary of the pipe. The optimization problem consists of finding the shape for the external boundary keeping the internal one fixed and minimizing the total area. The 4 design variables shown in Fig. 1 correspond with the coordinates of the points used to define the external boundary. Note that this example does not correspond to any real configuration, thus no units have been specified. Anyway, a coherent system of units has been used.

The maximum von Mises stresses in the model evaluated at boundary nodes have been restricted to $2.0 \times 10^{6}$.

It is well known that the optimal analytical geometry of the external boundary also corresponds to a circular shape. The following equations show the exact values of displacements and stresses for a given point with global Cartesian coordinates $(x, y)$, $k=R_{o} / R_{i}, r=\sqrt{x^{2}+y^{2}}$ and $\phi=\operatorname{arctg}(x / y)$, located in a thick-wall cylinder subjected to internal pressure. Hence, these equations can be used to evaluate the analytical solution of this optimization problem:

- Radial displacement:

$$
u_{r}=\frac{P(1+v)}{E\left(k^{2}-1\right)}\left[(1-2 v) r+R_{o}^{2} / r\right]
$$

- Stresses in cylindrical and Cartesian coordinates: 


$$
\begin{array}{rlrl}
\sigma_{r} & =\frac{P}{k^{2}-1}\left(1-R_{o}^{2} / r^{2}\right) & \\
\sigma_{t} & =\frac{P}{k^{2}-1}\left(1+R_{o}^{2} / r^{2}\right) & \sigma_{X X} & =\sigma_{r} \cdot \cos ^{2}(\phi)+\sigma_{t} \cdot \sin ^{2}(\phi) \\
\sigma_{Y Y} & =\sigma_{r} \cdot \sin ^{2}(\phi)+\sigma_{t} \cdot \cos ^{2}(\phi) \\
\sigma_{z} & =2 v \frac{P}{k^{2}-1} & \sigma_{X Y} & =\left(\sigma_{r}-\sigma_{t}\right) \cdot \sin (\phi) \cdot \cos (\phi)
\end{array}
$$

The external radius of the analytical solution for this problem ( $1 / 4$ of cross section) evaluated from (3) is $R_{o}=10.670330824461$. This corresponds to an area $A_{\text {opt }}=69.787307715081$.

\subsection{Evolutionary algorithm.}

For these numerical analyses the Differential Evolution (DE) algorithm has been used. DE is an evolutionary algorithm that has shown a robust performance yielding good results, even when applied to very different types of problems. DE was developed by Storn and Price [5]. Its key idea is the differential operator, which serves the same purpose as the crossover parameter in a standard genetic algorithm, namely to exchange information between parents when creating offspring. From the two different versions of the Differential Evolution algorithms proposed by Storn and Price the DE1 classical one has been used.

The initial values of the design variables and their allowed data range and geometrical constraints for the Pipe cross section problem are shown in Table 1.

Observe that constraint equations between the values of the design variables have been used in order to minimize the production of geometrically unfeasible individuals.

\subsection{Gradient-based algorithm.}

The deterministic optimization algorithm considered in this work defines the new values of the design variables for a new design using the results of the numerical analyses associated to the behavior of the existing design, and their sensitivity analysis. The methodology used in this paper corresponds to the algorithm developed by Navarrina [6]. Once the $k$-th design has been analyzed, the values for the design variables for the next design are defined as:

$$
\mathbf{v}_{k+1}=\mathbf{v}_{k}+\theta_{k} \mathbf{s}_{k}
$$

where $\mathbf{v}_{k}$ is the vector containing the values of the design variables for the $k$-th geometry, $\mathbf{v}_{k+1}$ is the vector corresponding to the next geometry, $\mathbf{s}_{k}$ is a unit vector defining the direction of change in the space of the design variables and $\theta_{k}$ is a scaling factor in this direction.

The algorithm computes the direction of change $\mathbf{s} k$ by using a SIMPLEX method with information coming from an exact first order sensitivity analysis of the OF and the constraints. Next, the scaling factor $\theta_{k}$ is computed by performing a line search minimization using second order directional sensitivity analysis.

\subsection{H-Adaptive Finite Element Analysis.}

An $h$-adaptive finite element analysis code has been used to obtain the values of the objective function and the degree of satisfaction of the constraints for each of the different geometrical configurations proposed by the optimization algorithms. The use 
of the $h$-adaptive strategy ensures the quality of the analysis providing FE solutions with a relative estimated discretization error in energy norm $\gamma$ lower than that specified by the analyst. The program uses the Zienkiewicz-Zhu error estimator in energy norm [7] to guide the $h$-adaptive analysis. The error estimator is based on the definition of the energy norm $\|\mathbf{u}\|$ :

$$
\|\mathbf{u}\|^{2}=\int_{\Omega} \boldsymbol{\sigma}^{T} \mathbf{D}^{-1} \boldsymbol{\sigma} d \Omega
$$

Hence, the following expression is used to evaluate the error estimation in energy norm for each element, $\eta_{e}$ :

$$
\eta_{e}^{2}=\int_{\Omega_{e}}\left(\boldsymbol{\sigma}^{*}-\boldsymbol{\sigma}_{h}\right)^{T} \mathbf{D}^{-1}\left(\boldsymbol{\sigma}^{*}-\boldsymbol{\sigma}_{h}\right) d \Omega
$$

where $\boldsymbol{\sigma}_{h}$ is the stress field directly obtained from the FE analysis, $\boldsymbol{\sigma}^{*}$ is a recovered improved stress field, $\mathbf{D}$ relates strains with stresses as $\sigma=\mathbf{D} \boldsymbol{\varepsilon}$ and $\Omega_{e}$ is the domain of element $e$.

Quadratic triangular elements have been used in the numerical examples. We have used the global least squares smoothing technique [7] to obtain the recovered stress field $\boldsymbol{\sigma}^{*}$ required by the Zienkiewicz-Zhu error estimator. The recovered stresses at the nodes along the boundary have been used to verify the stress constraints in the models. Other recovery techniques like the Superconvergent Patch Recovery technique (SPR) [8-10] or any other improvement of this recovery technique could also be used [11-14].

Cubic $B$-splines [15] have been used to define each of the geometrical configurations in terms of the coordinates of some definition points (parameterization).

In order to see the effect of the amount of discretization error contained in the analysis of each design over the behavior of the optimization algorithms (both evolutionary and deterministic) 6 different situations corresponding to different prescribed levels of the relative discretization error in energy norm $\gamma$ have been studied. The error values prescribed for the first 5 analyses were $1 \%, 2.5 \%, 5 \%, 10 \%$ and $20 \%$. In the $6^{\text {th }}$ case a $\gamma<100 \%$ tolerance has been specified, which in practical terms implies that the accuracy of the solution is not controlled and the number of elements is only depending on geometrical criteria (see examples in Fig. 2).

\section{4. $2 D$ model optimization using the evolutionary algorithm with in-house FEM code.}

Fig. 3 shows the effect of the prescribed maximum estimated relative error in energy norm $\gamma$ over the evolution of the area of the pipe cross section during each optimization process. It can be observed that, at least for this problem, the global aspect of the obtained optimum results for all the situations are quite similar.

However, as shown in Fig. 4, the effect of $\gamma$ over the evolution of the optimization process is especially significant if we compare the final results obtained with each different degree of the discretization error. Fig. 4 shows the evolution of the difference in relative terms between the area provided by the best individual obtained up to each generation and the area of the optimal analytical solution. The graph shows that the final solution associated to each analyses is significantly different from the analytical solution for the cases with higher values of the prescribed error $\gamma$, but approaches the exact solution for the lower values of $\gamma$. 
Fig. 5 represents the effect of $\gamma$ over the shape of the final solution provided by the optimization algorithm. It can be clearly observed that for high values of $\gamma$ the algorithm converges to shapes that are quite different from the optimal analytical solution and approaches the analytical solution for decreasing values of $\gamma$. The difference in area between the exact solution and that obtained for $\gamma=1 \%$ is only $0.38 \%$. In any case, one should take into account that the optimal analytical external contour (circular shape) cannot be exactly obtained because the $B$-splines used to define the boundary are unable to exactly reproduce a circular shape.

In the geometrical model, the external surface of the pipe has been imposed to be perpendicular to symmetry surfaces. When the error is not controlled or for the highest values of $\gamma$, the size of the elements is too big to capture this geometrical constraint, see highlighted area in Fig. 6.

On the other hand, it can be observed that, due to their size, the elements shown in Fig. 6 are unable to adequately evaluate the maximum level of stresses resulting from this geometry in this optimization problem. Therefore, individuals with geometries similar to that represented in Fig. 6 can be accepted as feasible, as shown in the first row of geometries in Fig. 5. With the most restrictive values of $\gamma$, the $h$-adaptive process will adequately adapt the mesh size to the local characteristics of the geometry, the stress levels will then be more accurately evaluated and this kind of geometries could be finally rejected.

In this problem, the optimization process tends to provide solutions with a value of the OF (area) lower than that of the analytical solution. This is due to the underestimation of the maximum value of stresses provided by the FEM for this problem. The level of this underestimation of stresses increases with the size of the elements used in the analysis. In order to quantify the quality of the solutions obtained with the different degrees of discretization error, we have performed a full $h$-adaptive analysis requiring a final estimated error in energy norm $\gamma<0.3 \%$ over each of the geometries displayed in Fig. 5. This has produced much more accurate approximations of the stress distributions in these geometries and, therefore, a much more accurate evaluation of the degree of satisfaction of the stress constraints. Table 2 shows the values of the cross sectional areas $A$ together with the difference with respect to the area of the analytical solution $A_{o p t}$ and the value of the maximum von Mises stress $\sigma_{v m}$ evaluated with $\gamma<0.3 \%$ together with their difference with respect to the maximum

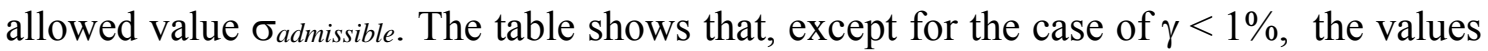
of the maximum von Mises stress were underestimated because of the lack of accuracy in the FE analyses. Therefore, these designs would not be valid as they would severely exceed the maximum allowed value for this magnitude. The table also shows that

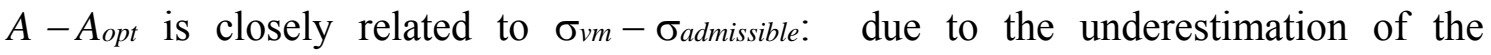
maximum von Mises stress the optimization algorithm reduces the area until this value reaches the specified limit. Hence, the optimization algorithm provides solutions with a lower area than those that would be obtained with an accurate evaluation of the maximum von Mises stress. Observe that the solution obtained with $\gamma \leq 1 \%$ is almost identical to the analytical solution, with a difference in areas of only $+0.38 \%$ and exceeding the allowable stresses by only $0.69 \%$.

For the sake of completeness this test case has been reproduced by linking the evolutionary algorithm with Ansys ${ }^{\circledR} 11[16]$, as this commercial package offers the possibility to run $h$-adaptive analyses both in $2 \mathrm{D}$ and $3 \mathrm{D}$. This code also uses the Zienkiewicz and Zhu error estimator [7] to guide the $h$-adaptive process, using a nodal 
averaging technique to obtain the recovered stress field $\boldsymbol{\sigma}^{*}$. Splines have been used in Ansys $^{\circledR}$ to define the geometrical configurations to be analyzed. Very similar results to those obtained with the in house code have been obtained.

\subsection{D model optimization using the gradient-based deterministic algorithm.}

The described 2D optimization test case has also been solved using the gradientbased algorithm. Fig. 7 shows a comparison between the convergence curves obtained with the different levels of prescribed discretization error for the case of the gradientbased deterministic algorithm. First, it can be seen that in all cases the convergence is much faster than with the evolutionary algorithm. Nevertheless, it can also be seen that after an initial quick drop of the OF, its evolution is different depending on the amount of the allowed discretization error.

During the first 10 iterations the convergence is not depending on the level of discretization error. This is because in these initial iterations the obtained designs are far away from the optimal one and the corresponding stress values are still far away from the restricted ones. Therefore, the constraints are not yet active and thus the process is still independent of the degree of accuracy in the evaluation of the constraints.

Fig. 8 shows the detailed evolution of the OF after the initial iterations. In this case, the evolution of the relative differences between the cross sectional area obtained at each iteration and the optimal analytical one is represented. It can be seen how a quick convergence to a practically exact value is obtained when $\gamma$ is fixed to $1 \%$. On the other hand, when higher values of $\gamma$ are employed, the evolution of the OF shows big oscillations and the process stops with final designs that, in fact, are not feasible because a more accurate analysis also shows too high values of the von Mises stress. The main reason of this behavior is the lack of reliability of the obtained values for the von Mises stress that drive all the optimization process. These values change every time the mesh is modified, even for a constant geometry, introducing a lot of "noise" in the convergence of the process and in the lack of reliability of the final design. This noise would not appear if a single mesh, conveniently adapted to each geometry, would have been used. In this case, this noise wouldn't be present and wouldn't produce oscillations, but the final optimum design wouldn't be reliable because the degree of accuracy of the computed von Mises stress would be very low.

Fig. 9 represents the effect of $\gamma$ over the final solution provided by the deterministic algorithm. As in the previous cases, it can be clearly observed that for high values of $\gamma$ the algorithm converges to shapes that are different from the optimal analytical solution, approaching to the analytical solution for decreasing values of $\gamma$. The difference in area between the exact solution and that finally obtained for $\gamma=1 \%$ is only $-0.15 \%$.

As in the case of the evolutionary algorithm, and in order to quantify the quality of the solutions obtained with the different degrees of discretization error, a full $h$-adaptive analysis requiring a final estimated error in energy norm $\gamma<0.3 \%$ was performed over each of the geometries displayed in Fig. 9. The results shown in Table 3 are similar to those previously shown for the evolutionary algorithm: the optimal solutions provided by the optimization process underestimate the maximum value of the von Mises stress that would be obtained with more accurate FE models. Solutions with a lower area than those that would be obtained with an accurate evaluation of the maximum von Mises stress are, thus, obtained. However, the results obtained in this case are more accurate than those obtained with the evolutionary algorithm. The solution obtained with $\gamma \leq 1 \%$ 
is again quite close to the analytical solution, with a difference in areas of $-0.15 \%$ and exceeding the allowable stresses by only $0.33 \%$.

\subsection{D model optimization using the evolutionary algorithm and Ansys ${ }^{\circledR} 11$.}

A three-dimensional version of this test case has also been solved with the evolutionary algorithm using the model represented in Fig. 10 which uses 12 design variables to represent the geometry together with the appropriate symmetry boundary conditions. As in the $2 \mathrm{D}$ case, the analytical optimal external radius is $R_{o}=10.670330824461$ for an internal radius $R_{i}=5$. The length of the model along the axis of the pipe was $L=20$, therefore, the volume of the optimal geometry is $V_{o p t}=1395.74615$. The optimization loop has started from an external cylindrical surface of radius 12 .

In this case only three error levels have been prescribed: $5 \%, 10 \%$ and $\gamma<100 \%$ (no error control). The initial global element size has been set to 4 units in all cases. As the stopping criterion, we considered that the optimization problem reached convergence when the solution did not improve after 150 consecutive generations. The volume evolution and the percentage of error in volume with respect to the analytical solution have been represented in Fig. 11 and Fig. 12.

The optimization process has required 513 generations for $\gamma<5 \%, 589$ for $\gamma<10 \%$ and 927 when the discretization error has not been controlled. This shows that, in this case, the lack of control on the accuracy of the FE results has a negative effect over the convergence of the process to a final solution.

Note that the curves used to define the external surface of the model (see Fig. 10) are splines. As these curves are unable to reproduce a circumference, the model used in the analyses is unable to exactly reproduce the optimal analytical geometry. This explains why the results provided by the optimization algorithm seem to converge to solutions that are not exactly the optimal analytical solution.

Fig. 13 shows the final geometries obtained with the optimization algorithm and their comparison with the optimal analytical solution. It can be clearly observed that decreasing the value of $\gamma$ produces geometries that are closer to the optimal analytical solution. It can also be observed that, when the discretization error is not controlled, the solution found for 927 generations does not seem to be better than that for 500 generations; in fact we will demonstrate that it is worst.

As in the $2 \mathrm{D}$ case, in order to obtain a more accurate evaluation of the maximum value of the von Mises stresses in the final solutions provided by the optimization processes, the geometries displayed in Fig. 13 have been analyzed requiring a final estimated relative error in energy norm $\gamma \leq 0.8 \%$. The results obtained are displayed in Table 4. This table shows that when the discretization error is not controlled, the maximum value of the von Mises stress is not accurately evaluated, resulting in a considerable underestimation of the stress level that guides the optimization process to a non valid final solution. In fact, the last column in the table shows that when the discretization error is not controlled, the lack of accuracy in the evaluation of the stresses induced the generation of a solution after 927 generations, at the end of the optimization process, that is worst than that obtained after 500 . As in the $2 \mathrm{D}$ case, the table shows that the solutions obtained with error control are more accurate for more restrictive values of $\gamma$. 
The optimal analytical solution corresponds to an external cylindrical surface of radius $R_{o}=10.670330824461$. Considering this, and taking into account that the geometrical variables (see Fig. 10) define the radial distances $r_{i}$ of the $n$ points $(n=9)$ used to define the external surface, we defined an indicator $\Phi$ of the quality of the solution as:

$$
\Phi=\frac{100}{R_{o}} \frac{\sum_{i=1}^{n}\left|r_{i}-R_{o}\right|}{n}
$$

This indicator is a measure of the error in radius with respect to the optimal analytical solution expressed as a percentage of $R_{o}$. This is a more meaningful indicator of the quality of the solution than the volume itself, as $\Phi$ is a measure of the quality of the shape of the solution. In the analytical solution $r_{i}=R_{o}$, thus $\Phi=0$. Therefore the best solutions would be those with the lowest values of $\Phi$. The evolution of $\Phi$ in the case in which the error is not controlled has been represented in Fig. 14.

Both, Fig. 11 and Fig. 12, apparently show that the optimization process has always improved the solution and thus that, when the error is not controlled, the solution at the end of the optimization process, after 927 generations, is better than the solution at 500 generations. However, the decrease in volume is not followed by a decrease in the indicator $\Phi$. In fact, as shown in Table 4, the solution obtained after 927 generations was found to be worse than the solution obtained after 500 generations.

\section{EVOLUTIONARY ALGORITHMS WITH MESH GENERATION BY PROJECTION OF THE DISCRETIZATION ERROR}

The results presented in the previous section have clearly demonstrated that if a minimum quality of the results used to drive structural shape optimization processes is not ensured, the optimization process will not converge to the optimal solution, providing solutions that could notably violate the satisfaction of the constraint equations. However, the use of $h$-adaptive techniques to control the quality of the finite element analyses implies an additional high computational cost as each geometrical configuration must be evaluated a number of times until the prescribed level of accuracy is reached. Due to the high number of different shape configurations to be analyzed, the use of this kind of techniques can be critical when evolutionary algorithms are considered. For example, in the 3D problem shown in Section 2.6, the ratio of the mean time required for the evaluation of each individual for $\gamma \leq 10 \%$ to the mean time required with no error control was 1.8. This ratio reached the value of 2.7 for $\gamma \leq 5 \%$.

In the cases where evolutionary algorithms are used, and in order to reduce the computational cost associated to the creation of an adapted mesh for each of the individuals to be analyzed, we propose the use of the technique described by Bugeda et al.[1] that will be summarized below. The origin of this technique, and its application to deterministic optimization algorithms, can be seen in references $[2,3]$. In the present work we have adapted this technique for evolutionary optimization algorithms. 


\subsection{Algorithm for direct definition of h-adapted meshes for all the individuals of a generation.}

For each generation of individuals the proposed strategy (see a graphical representation of the algorithm in Fig. 15) is summarized as follows:

1.- Selection of the reference individual. A specific individual is selected as reference individual for the generation. This individual can be fixed at the very beginning of the optimization process as an initial design. Nevertheless, the best results have been obtained by defining a different reference individual for each generation of the optimization process using the mean values of the design variables of all the individuals of that particular generation. Thus, if we have a population with $P$ individuals, the values of $\mathbf{v}_{r}$ that define the reference one are computed as

$$
\mathbf{v}_{r}=\frac{1}{P} \sum_{p=1}^{P} \mathbf{v}_{p}
$$

where $\mathbf{v}_{p}$ indicates the values of the design variables $\mathbf{v}$ corresponding to the $p^{\text {th }}$ individual using the selected parameterization.

2.- H-adadptive analysis of the reference individual. The reference individual for the generation is analyzed using a traditional finite element approach, including an adaptive remeshing strategy to ensure a good quality of the results.

3.- Sensitivity analysis of the reference individual. Once a good mesh is obtained for the analysis of the reference individual, a complete sensitivity analysis of all the magnitudes involved in the adaptive remeshing strategy is executed. This includes the evaluation of the sensitivity of the discretization error, see (6), for each element in the mesh. The following equation was derived in reference [2] to evaluate this magnitude.

$$
\begin{aligned}
\frac{\partial\left(\eta_{e}\right)^{2}}{\partial v_{i}}= & \int_{\Omega_{\xi}}\left[\left(\frac{\partial \boldsymbol{\sigma}^{*}}{\partial v_{i}}-\frac{\partial \boldsymbol{\sigma}_{h}}{\partial v_{i}}\right)^{T} \mathbf{D}^{-1}\left(\boldsymbol{\sigma}^{*}-\boldsymbol{\sigma}_{h}\right)+\right. \\
& \left(\boldsymbol{\sigma}^{*}-\boldsymbol{\sigma}_{h}\right)^{T} \frac{\partial \mathbf{D}^{-1}}{\partial v_{i}}\left(\boldsymbol{\sigma}^{*}-\boldsymbol{\sigma}_{h}\right)+ \\
& \left(\boldsymbol{\sigma}^{*}-\boldsymbol{\sigma}_{h}\right)^{T} \mathbf{D}^{-1}\left(\frac{\partial \boldsymbol{\sigma}^{*}}{\partial v_{i}}-\frac{\partial \boldsymbol{\sigma}_{h}}{\partial v_{i}}\right)+ \\
& \left.\left(\boldsymbol{\sigma}^{*}-\boldsymbol{\sigma}_{h}\right)^{T} \mathbf{D}^{-1}\left(\boldsymbol{\sigma}^{*}-\boldsymbol{\sigma}_{h}\right) \operatorname{tr}\left(\mathbf{J}^{-1} \frac{\partial \mathbf{J}}{\partial v_{i}}\right)\right]|\mathbf{J}| d \Omega_{\xi}
\end{aligned}
$$

Where $\Omega_{\xi}$ is the element domain in local coordinates and $\mathbf{J}$ is the Jacobian matrix corresponding to the transformation of coordinates.

Fuenmayor et al. [4] extended the Zienkiewicz-Zhu error estimator [7] to shape sensitivity analysis in order to develop a discretization error estimator for shape sensitivity analysis, obtaining the expression shown in (9). This proves that the sensitivity of the error is equivalent to the error in sensitivities.

These calculations require the evaluation of the sensitivities of the nodal coordinates, which describes how the mesh evolves when the design variables change. For nodes located on the boundary of the domain these sensitivities can be easily obtained from the first order sensitivity analysis of the $B$-spline curves 
used to define the boundary. A description and comparison of methods commonly used to evaluate the sensitivities of the internal nodes can be found in [17]. The classical Laplacian smoothing technique has been used in the numerical examples presented in this work.

The evaluation of $\partial \eta_{e}^{2} / \partial x_{i}$ also requires the evaluation of the recovered stress field $\boldsymbol{\sigma}^{*}$. Although other procedures for nodal stress recovery, such as the SPR technique $[8,9]$, could be used, in this work stresses are recovered using a global least squares smoothing technique [7].

4.- Projection of magnitudes used in the h-adaptive process. For each of the individuals to be analyzed, the values of all magnitudes involved in the adaptive remeshing strategy evaluated in the previous step are projected from the reference, using the corresponding sensitivity analysis. The magnitudes to be projected are the nodal coordinates ( $x$ and $y$ ), the error estimator in energy norm $(\eta)$ and the energy norm $(\|\mathbf{u}\|)$. The following expressions are used in these projections:

$$
\begin{gathered}
(x, y)_{p}=(x, y)_{r}+\sum_{i}^{n}\left(v_{p_{i}}-v_{r_{i}}\right)\left(\frac{\partial x}{\partial v_{i}}, \frac{\partial y}{\partial v_{i}}\right) \\
\eta_{p}^{2}=\eta_{r}^{2}+\sum_{i}^{n}\left(v_{p_{i}}-v_{r_{i}}\right) \frac{\partial \eta^{2}}{\partial v_{i}} \\
\|\mathbf{u}\|_{p}^{2}=\|\mathbf{u}\|_{r}^{2}+\sum_{i}^{n}\left(v_{p_{i}}-v_{r_{i}}\right) \frac{\partial\|\mathbf{u}\|^{2}}{\partial v_{i}}
\end{gathered}
$$

where sub-indexes $r$ and $p$ are respectively related to the reference individual and the individual to which the information is projected, and $v_{i}$ are the design variables used to define the geometry, see [1].

This projection provides, without any additional computation, an approximation to the values that would be obtained for each specific individual if it would have been computed with the same finite element mesh used for the reference individual, thus providing the necessary information to perform an adaptive remeshing over the next design, even before any new computation is performed.

5.- Generation of the h-adapted mesh. An adapted mesh is generated for each individual using the projected values of the last step. This $h$-adapted mesh is used for the evaluation of the objective function and the restrictions of the individual.

The generation of every new mesh in the remeshing procedure requires the definition of a mesh optimality criterion. In this work a mesh is considered as optimal when the error density is equally distributed across the volume, see [3].

Fig. 22 in next section will show an example of analysis of a generation of individuals. 


\section{NUMERICAL EXAMPLES}

This section shows three optimization examples solved using the proposed strategy.

\subsection{Pipe cross section.}

We used our own FE code for the structural analysis of each of the geometries proposed by the optimization algorithm to solve again the 2D case of the Pipe example described in Section 2. The technique described in Section 3 was used for the direct definition of $h$-adapted meshes for the individuals analyzed in each generation. The maximum admissible relative error in energy norm for each of the analysis was set to $\gamma=1 \%$. 150 generations with 30 individuals per generation were considered. The results showed that in the $97 \%$ of the 4500 individuals analyzed, the use of the proposed technique [1] directly provided the appropriate mesh for the FE analysis. This implies a considerable reduction of the computational cost associated to the $h$-adaptive analyses.

\subsection{Hook}

The second numerical example is the optimization of the shape of a hook in order to minimize its weight. Note that this example is a modification of that presented in [1], using a different load and a different prescribed error level. Therefore the results here obtained cannot be directly compared with those in [1]. The initial shape, the applied load and the geometry definition points (hollowed dots) are shown in Fig. 16. The coordinates of 19 points are used as design variables, 16 of them can move horizontally, one can move vertically and the rest have been enforced to move along straight lines inclined $45^{\circ}$. The resulting load applied over the inner part of the hook is $6300 \mathrm{~N}$. The material properties are $E=21000000 \mathrm{~N} / \mathrm{cm}^{2}$ and $v=0.3$. A plane stress model has been used. The objective function is the total cross sectional area of the hook. The maximum von-Mises stresses along the boundary of the model are restricted to $20000 \mathrm{~N} / \mathrm{cm}^{2}$. The minimum thickness of the spike of the hook is limited to $0.5 \mathrm{~cm}$.

20 individuals per generation were used in the analysis. The optimization process needed a total of 400 generations. A maximum estimated relative error in energy norm $\gamma \leq 2.0 \%$ was prescribed.

The shape provided by the optimization algorithm, which has an area of $88.71 \mathrm{~cm}^{2}$, has also been represented in Fig. 16 for comparison. It can be observed that the optimization process displaces the spike of the hook so that it coincides with the resultant of the load forces in order to eliminate the bending moment over this region.

Fig. 3 showed that the results during the initial steps of the optimization process are not significantly affected by the prescribed accuracy of the analyses. This has allowed implementing a very simple technique that reduces the computational cost of the optimization. This technique simply consists of defining the value of the admissible estimated error in energy norm $\gamma$ as a function of the generation number, decreasing the value of $\gamma$ during the progress of the optimization process. This technique considerably reduces the computational cost associated to the analysis of the first generations of individuals without affecting the accuracy of the results corresponding to the last steps of the optimization process. The evolution of the prescribed error along the optimization process, that only considers the required accuracy of $\gamma=2 \%$ after generation 150 , is represented in Fig. 17. An exponential reduction from $\gamma=20 \%$ to $\gamma=2 \%$ in the first 150 generations has been considered to solve this problem. 
Fig. 18 shows examples of the different mesh densities used along the optimization process. Observe that the optimization algorithm is able to provide reasonable approximations to the final geometry in the early stages of the process using coarse meshes (see geometries for generations 6 and 57) that require a low computational cost. Fig. 19 shows the evolution of the best individual found along the optimization process.

\subsection{Gravity dam.}

The shape optimization of the gravity dam represented in Fig. 20 has been solved. Both dead weight and water hydrostatic pressure have been considered in the analyses. The model does not include any sharp reentrant corner that could produce stress singularities masking the evolution of the adaptive remeshing processes. The shape optimization problem consisted of running the genetic algorithm for a total 100 generations, with 30 individuals per generation, to improve the shape of the internal boundary in order to reduce the cross sectional area of the gravity dam, keeping fixed the external boundary. A total of 7 points have been used to define the internal boundary. As indicated in Fig. 21, the coordinates of 5 of these points have been considered as the design variables for this problem. The maximum von-Mises stresses along the boundary of the model have been restricted to $2.75 \cdot 10^{6} \mathrm{~Pa}$.

A maximum estimated relative error in energy norm $\gamma=2.5 \%$ has been required for the analysis of the final solution.

Fig. 22 shows an example of analysis of a generation of individuals. The reference individual is displayed at the top of the figure. In this case, the error projection technique described in Section 3 directly provides the appropriate mesh for the analysis of 29 of the individuals evaluated in this generation. Only one of the individuals has required a further remeshing step in order to reduce the discretization error below the specified value.

As in the previous case we used an exponential function to define the reduction of $\gamma$. In this case we reduced prescribed error from an initial value $\gamma=20 \%$ to $\gamma=2.5 \%$ in 100 generations, see Fig. 23. For comparison purposes, we also considered a second test in which the prescribed error was set to $\gamma \leq 2.5 \%$ during all the optimization process, see Fig. 23. The use of the error projection technique described in Section 3 has provided the appropriate mesh for the FE analysis of $95 \%$ of the individuals considered in the optimization process for both cases.

Fig. 24 shows the evolution of the objective function (area) during the optimization process. This figure clearly shows that the use of high values of $\gamma$ at the beginning of the process and its smooth reduction have provided similar evolutions of the best individual along the optimization process and similar area reductions after 100 generations (from $2984.9 \mathrm{~m}^{2}$ to $2527 \mathrm{~m}^{2}$ in the case of the constant error level and to $2544 \mathrm{~m}^{2}$ in the case of the variable error level). The differences shown in Fig. 24 are reasonable taking into account the nature of the evolutionary algorithm and that the optimization processes have not yet converged after 100 iterations.

Fig. 25 shows the difference between the original and the similar optimized designs provided by both analyses.

Note that the thin section on the downstream face of the dam is obtained because the only constraint considered in the optimization process was the maximum value of the von-Mises stresses along the boundary of the model. A stability constraint should also be imposed for a more realistic analysis. 
The previous results show that the technique consisting on the gradual reduction of $\gamma$ is a valid technique to reduce the computational cost of the optimization process.

When defining the reduction of $\gamma$ the analyst must take into account that if $\gamma$ decreases too fast then there will only be a small reduction of the computational cost. On the other hand, if $\gamma$ reduces slowly then the optimization process could prematurely converge to solutions that would not satisfy the constraints if analyzed with the final prescribed value of $\gamma$. The reduction of $\gamma$ should be somehow coupled to the evolution of the optimization process, which depends on the number of design variables and other parameters like those used to control the optimization algorithm. How this coupling must be achieved is still an open question that will be a matter of our research in the near future.

\section{CONCLUSIONS}

This paper has shown that a minimum quality of the results used to drive structural shape optimization processes must be ensured; otherwise converge to the optimal solution can be prevented, and solutions that notably violate the satisfaction of the constraint equations can be obtained. Therefore, the optimization processes that make use of the finite element method to evaluate the objective function and the degree of satisfaction of the constraints require the use of error control techniques, preferably using adaptive remeshing techniques, as it is well known that the cheapest mesh for producing a solution with a fixed quality at minimum cost is an adapted one.

Due to the high number of individuals to be analyzed, the computational cost associated to the use of adaptive analysis techniques can be critical when evolutionary algorithms are considered. For this kind of optimization algorithms we propose the use of the technique for the $h$-adaptive analysis of generations of individuals presented by Bugeda et al.[1]. This technique uses the projection of the estimated discretization error in energy norm to obtain the meshes required for the analysis of the individuals considered in each generation. The presented results show the efficiency of this technique which, in the vast majority of cases, provided the appropriate $h$-adapted mesh for the FE analyses with an error level lower than that specified by the analyst, thus avoiding the full adaptive remeshing process.

Preliminary results have shown that the maximum discretization error level imposed over the FE analyses has no significant effect over the first steps of the optimization process. Hence, we have proposed the use of a technique where the maximum discretization error level decreases with the progress of the optimization process. The technique is very simple and can be used with any FE code with adaptive analysis capabilities. Despite of its simplicity, the main advantage of this technique is that its adequate use considerably reduces the computational cost associated to the analysis of the first generations of individuals without affecting the accuracy of the results corresponding to the last steps of the optimization process. This requires the reduction of $\gamma$ to be adequately coupled to the evolution of the optimization process. Further research is necessary to establish how this coupling can be achieved. 


\section{ACKNOWLEDGMENTS}

The first and third authors are grateful for the financial support received for the development of this paper through the research project DPI2007-66773-C02-01 of the Ministerio de Educación y Ciencia (Spain) and the funding received from the Generalitat Valenciana and the Universidad Politécnica de Valencia.

The second author is grateful for the financial support received for the development of this paper through the research project DPI2008-05250 of the Ministerio de Ciencia e Innovación (Spain).

The authors also want to thank MsC Santos López-Real for his help with the use of evolutionary algorithms.

\section{REFERENCES}

1. Bugeda G, Ródenas JJ, Oñate E. An Integration of a Low Cost Adaptive Remeshing Strategy in the Solution of Structural Shape Optimization Problems using Evolutionary Methods. Computers \& Structures 2008; 86:1563-1578. DOI: 10.1016/j.compstruc.2007.05.010.

2. Bugeda G, Oliver J. A General Methodology for Structural Shape Optimization Problems using Automatic Adaptive Remeshing. International Journal for Numerical Methods in Engineering 1993; 36:3161-3185. DOI: 10.1002/nme.1620361807.

3. Bugeda G, Oñate E. A Methodology for Adaptive Mesh Refinement in Optimum Shape Design Problems. Computing Systems in Engineering 1994; 5:91-102.

4. Fuenmayor FJ, Oliver JL, Ródenas JJ. Extension of the Zienkiewicz-Zhu Error Estimator to Shape Sensitivity Analysis. International Journal for Numerical Methods in Engineering 1997; 40:14131433.

5. R. Storn and K. Price. (1995, March 1995). Differential evolution - A simple and efficient adaptive scheme for global optimization over continuous space. International Computer Science Institute, Berkeley, CA, USA. Available: ftp://ftp.icsi.berkeley.edu/pub/techreports/1995/tr-95-012.pdf

6. F. Navarrina, "Una metodología general para optimización estructural en diseño asistido por ordenador," 1987.

7. Zienkiewicz OC, Zhu JZ. A Simple Error Estimation and Adaptive Procedure for Practical Engineering Analysis. International Journal for Numerical Methods in Engineering 1987; 24:337357.

8. Zienkiewicz OC, Zhu JZ. The Superconvergent Patch Recovery and a Posteriori error Estimates. Part I: The Recovery Technique. International Journal for Numerical Methods in Engineering 1992; 33:1331-1364.

9. Zienkiewicz OC, Zhu JZ. The Superconvergent Patch Recovery and a Posteriori Error Estimates. Part II: Error Estimates and Adaptivity. International Journal for Numerical Methods in Engineering 1992; 33:1365-1382.

10. Zienkiewicz OC, Zhu JZ, Wu J. Superconvergent Patch Recovery Techniques - some further Tests. Communications in Numerical Methods in Engineering 1993; 9:251-258. DOI: 10.1002/cnm.1640090309.

11. Díez P, Ródenas JJ, Zienkiewicz OC. Equilibrated Patch Recovery Error Estimates: Simple and Accurate Upper Bounds of the Error. International Journal for Numerical Methods in Engineering 2007; 69:2075-2098. DOI: 10.1002/nme.1837.

12. Ródenas JJ, Tur M, Fuenmayor FJ, Vercher A. Improvement of the Superconvergent Patch Recovery Technique by the use of Constraint Equations: The SPR-C Technique. International Journal for Numerical Methods in Engineering 2006; 70:705-727. DOI: 10.1002/nme.1903.

13. Wiberg NE, Abdulwahab F. Patch Recovery Based on Superconvergent Derivatives and Equilibrium. International Journal for Numerical Methods in Engineering 1993; 36:2703-2724. 
14. Wiberg NE, Abdulwahab F, Ziukas S. Enhanced Superconvergent Patch Recovery Incorporating Equilibrium and Boundary Conditions. International Journal for Numerical Methods in Engineering 1994; 37:3417-3440.

15. Faux ID, Pratt MJ, Computational Geometry for Design and Manufacture. Chichester : Ellis Horwood: 1987.[5th. reprint.] ed.

16. Ansys ${ }^{\circledR}$ Release 11.0, Help System, ANSYS Inc. , 2007.

17. Ródenas JJ, Fuenmayor FJ, Tarancón JE. A Numerical Methodology to Assess the Quality of the Design Velocity Field Computation Methods in Shape Sensitivity Analysis. International Journal for Numerical Methods in Engineering 2004; 59:1725-1747. 


\begin{tabular}{cccc}
\hline \hline Design variable & Initial value & Range & Constraints \\
\hline$V_{1}$ & 20 & {$[5.2-50.0]$} & \\
$V_{2}$ & 19 & {$[4.0-50.0]$} & \\
$V_{3}$ & 19 & {$[4.0-50.0]$} & $V_{3}<V_{1}-0.5$ \\
$V_{4}$ & 20 & {$[5.2-50.0]$} & $V_{4}>V_{2}+0.5$ \\
\hline \hline
\end{tabular}

Table 1. Pipe cross section. Values of design variables. 


\begin{tabular}{ccccc}
\hline \hline$\gamma$ & $A$ & $A-A_{\text {opt }}$ & $\sigma_{v m}$ & $\sigma_{v m}-\sigma_{\text {admissible }}$ \\
\hline No control & 57.22 & $-18.00 \%$ & 2222330 & $11.12 \%$ \\
$20 \%$ & 57.52 & $-17.58 \%$ & 2229070 & $11.45 \%$ \\
$10 \%$ & 58.64 & $-15.97 \%$ & 2265360 & $13.27 \%$ \\
$5 \%$ & 63.94 & $-8.39 \%$ & 2074650 & $3.73 \%$ \\
$2.5 \%$ & 68.74 & $-1.50 \%$ & 2025850 & $1.29 \%$ \\
$1 \%$ & 70.05 & $0.38 \%$ & 2013710 & $0.69 \%$ \\
\hline \hline
\end{tabular}

Table 2. 2D Model. Evolutionary algorithm. Influence of $\gamma$ over the accuracy of the objective function and the degree of satisfaction of stress constraint equations (maximum von Mises stress of final geometries evaluated using $\gamma=0.3 \%)$. 


\begin{tabular}{ccccc}
\hline \hline$\gamma$ & $A$ & $A-A_{\text {opt }}$ & $\sigma_{v m}$ & $\sigma_{v m}-\sigma_{\text {admissible }}$ \\
\hline No control & 63.10 & $-9.58 \%$ & 2039197 & $1.96 \%$ \\
$20 \%$ & 64.05 & $-8.22 \%$ & 2044204 & $2.21 \%$ \\
$10 \%$ & 62.77 & $-10.06 \%$ & 2065720 & $3.29 \%$ \\
$5 \%$ & 67.66 & $-3.05 \%$ & 2031427 & $1.57 \%$ \\
$2.5 \%$ & 67.15 & $-3.78 \%$ & 2021792 & $1.09 \%$ \\
$1 \%$ & 69.68 & $-0.15 \%$ & 2006560 & $0.33 \%$ \\
\hline \hline
\end{tabular}

Table 3. 2D model. Deterministic algorithm. Influence of $\gamma$ over the accuracy of the objective function and the degree of satisfaction of stress constraint equations (maximum von Mises stress of final geometries evaluated using $\gamma=0.3 \%$ ) 


\begin{tabular}{cccccc}
\hline \hline$\gamma$ & Generation & $V$ & $V-V_{\text {opt }}$ & $\sigma_{v m}$ & $\sigma_{v m}-\sigma_{\text {admissible }}$ \\
\hline $5 \%$ & 513 & 1440.53 & $3.21 \%$ & 1996000 & $-0.200 \%$ \\
$10 \%$ & 589 & 1438.85 & $3.09 \%$ & 2006700 & $0.335 \%$ \\
No control & 500 & 1454.95 & $4.24 \%$ & 2033700 & $1.685 \%$ \\
No control & 927 & 1432.25 & $2.62 \%$ & 2069900 & $3.495 \%$ \\
\hline \hline
\end{tabular}

Table 4. 3D model. Evolutionary algorithm + Ansys ${ }^{\circledR}$. Influence of $\gamma$ over the accuracy of the objective function and the degree of satisfaction of stress constraint equations (maximum von Mises stress $\sigma v m$ of final geometries evaluated using $\gamma \leq 0.8 \%$ ) 


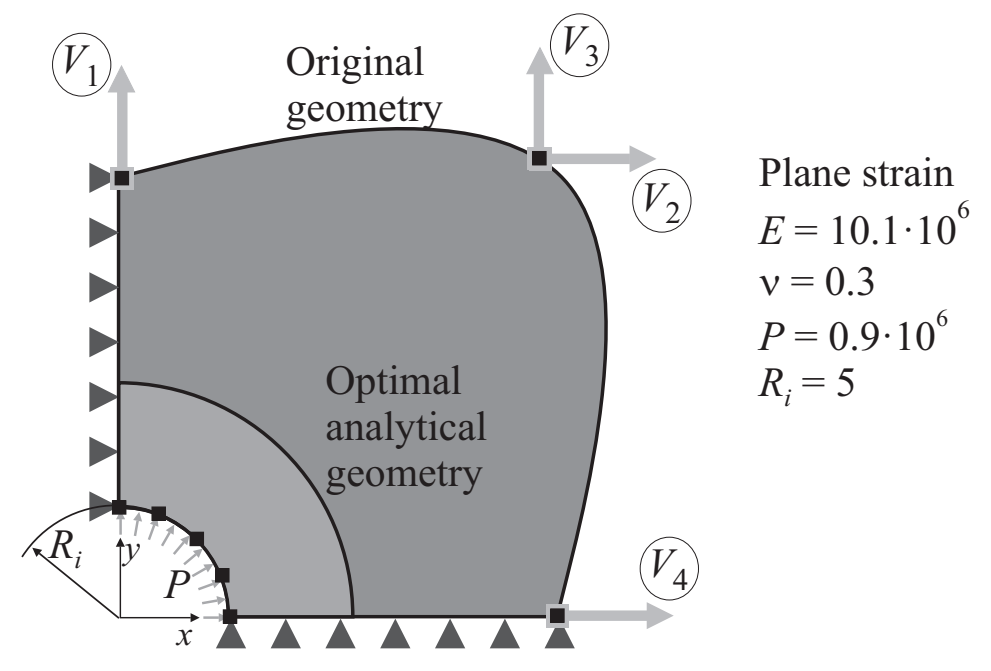

Fig. 1. Pipe cross section. Analysis data, original model, optimal analytical solution and design variables. 

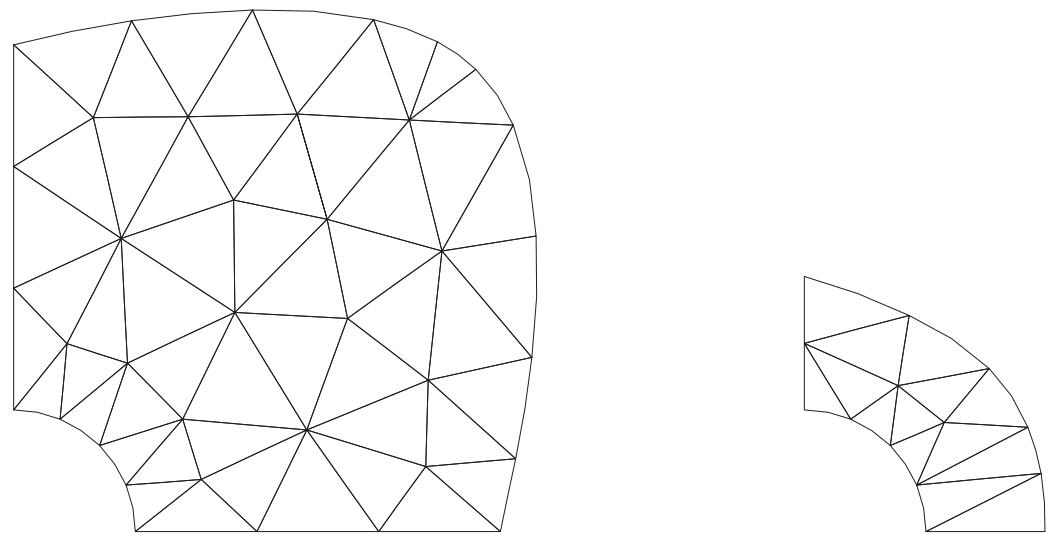

Fig. 2. Examples of initial meshes before any adaptive operation. 


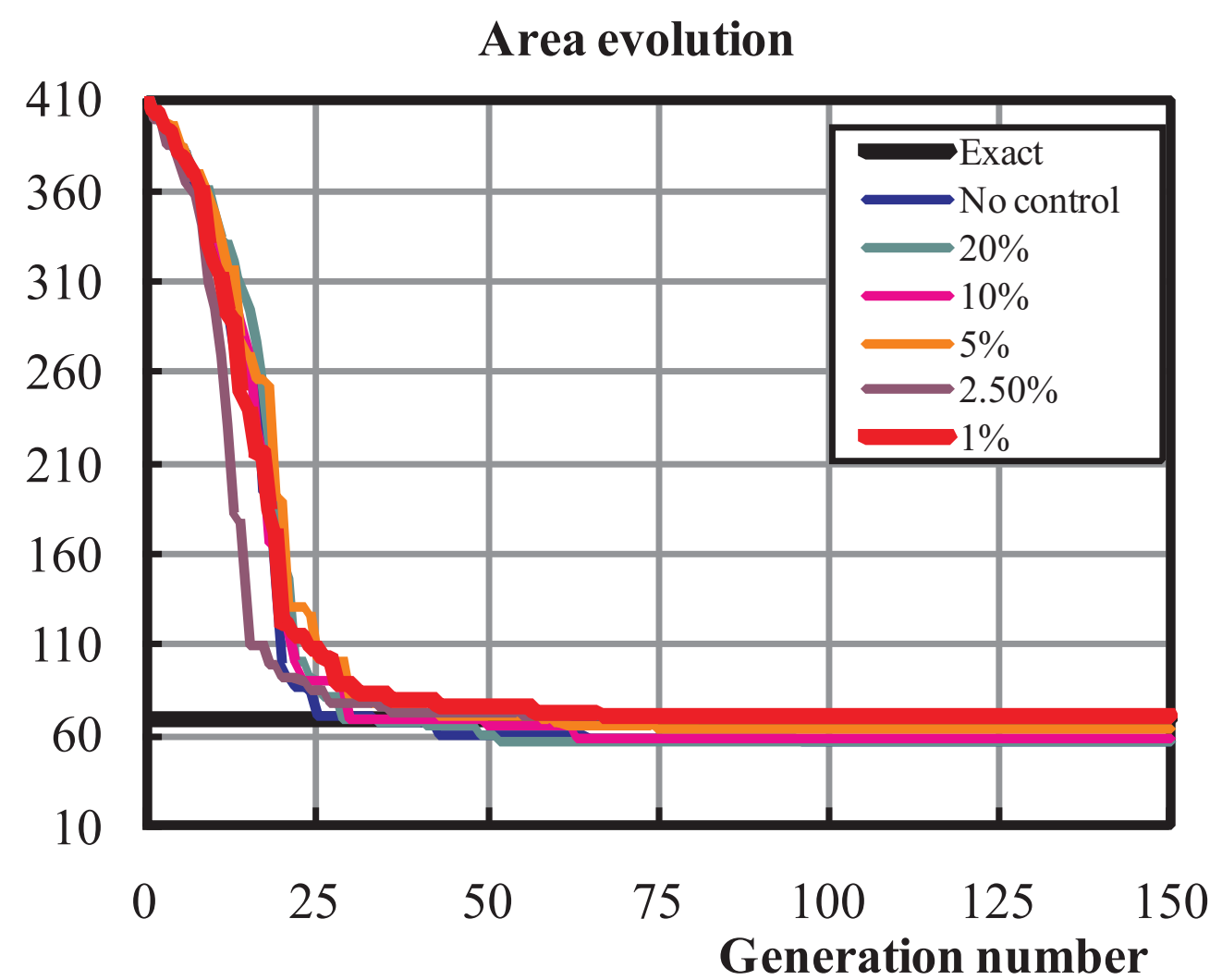

Fig. 3. 2D model. Evolutionary algorithm. Influence of $\gamma$ over the evolution of the objective function. 


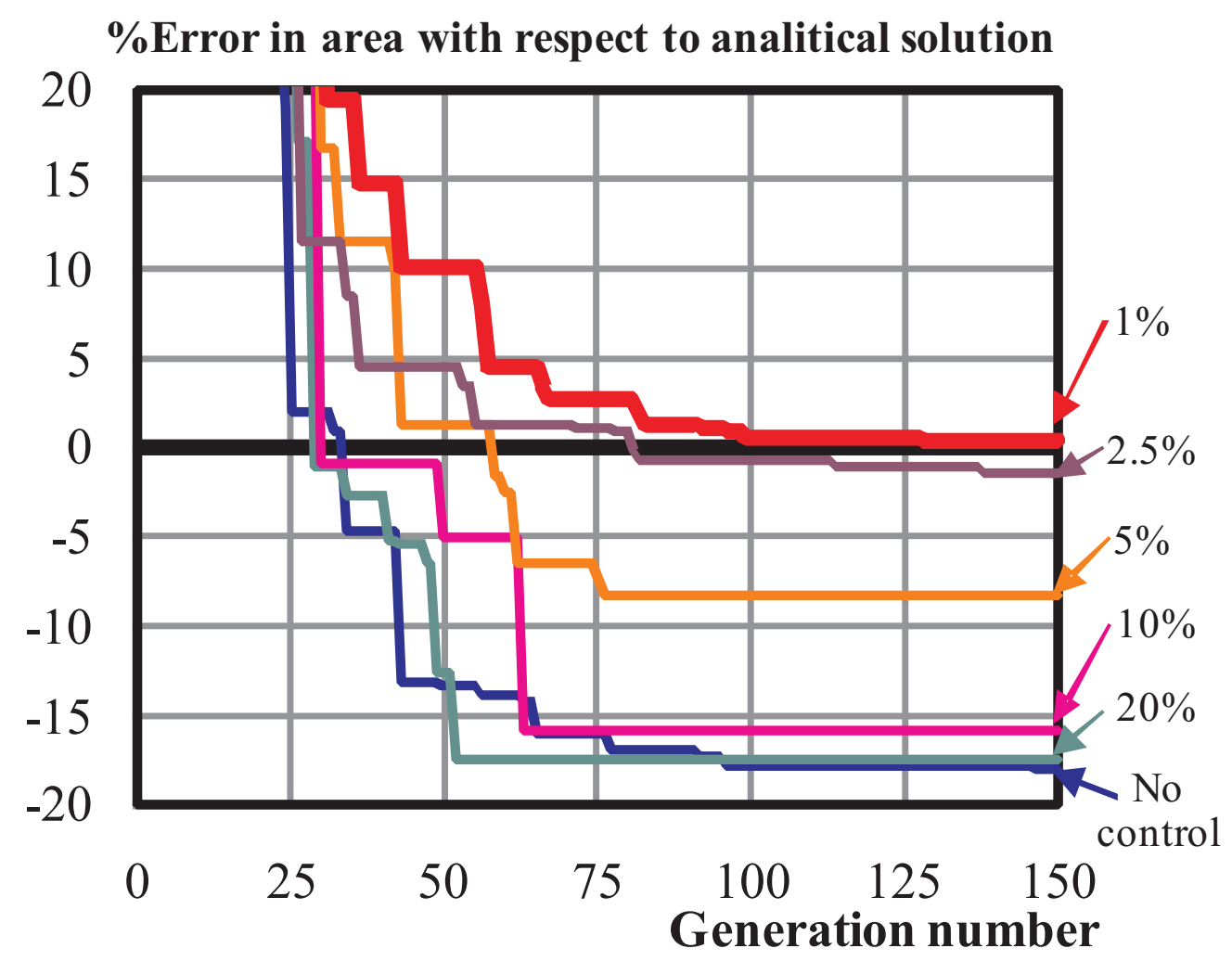

Fig. 4. 2D model. Evolutionary algorithm. Influence of $\gamma$ over the evolution of the error in the objective function with respect to the analytical solution. 

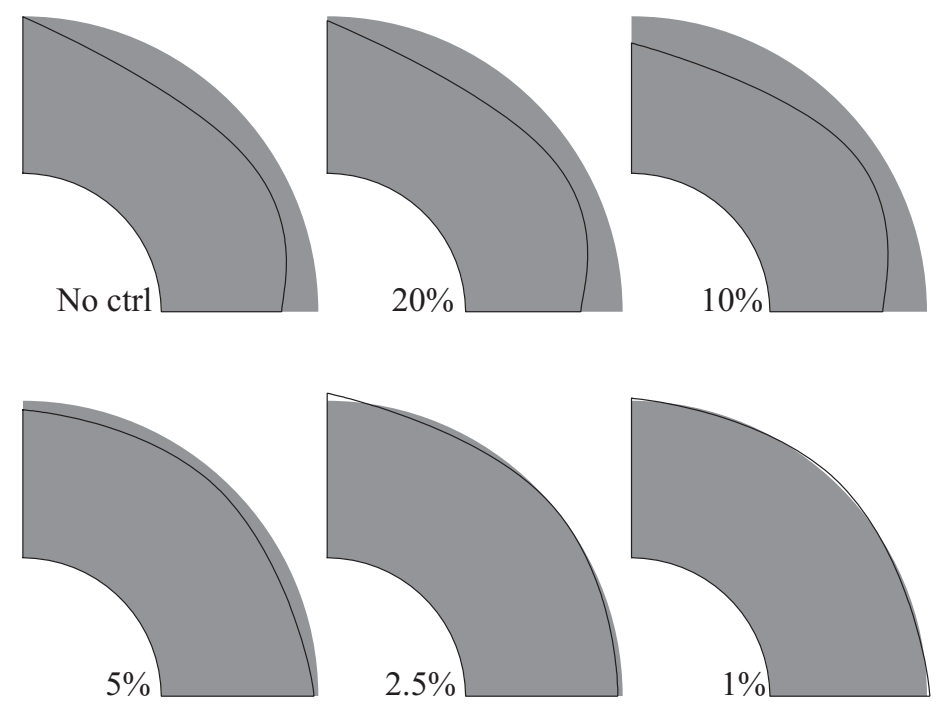

Fig. 5. 2D model. Evolutionary algorithm. Influence of $\gamma$ over the optimal solution found (black contour) and comparison with optimal analytical solution (shaded area). 


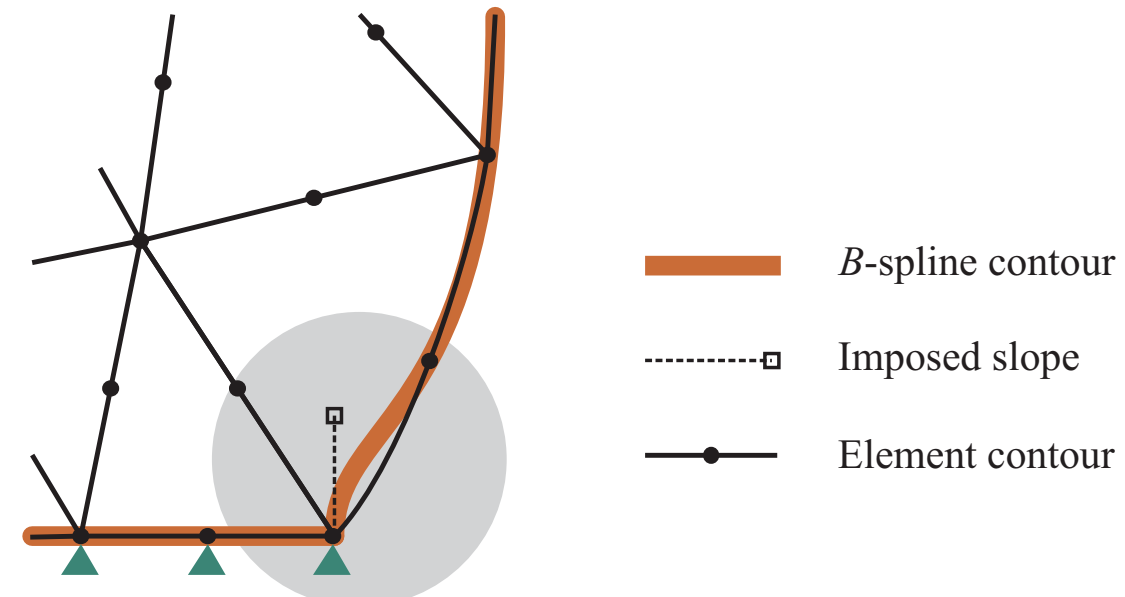

Fig. 6. Geometrical constrain and mesh. 


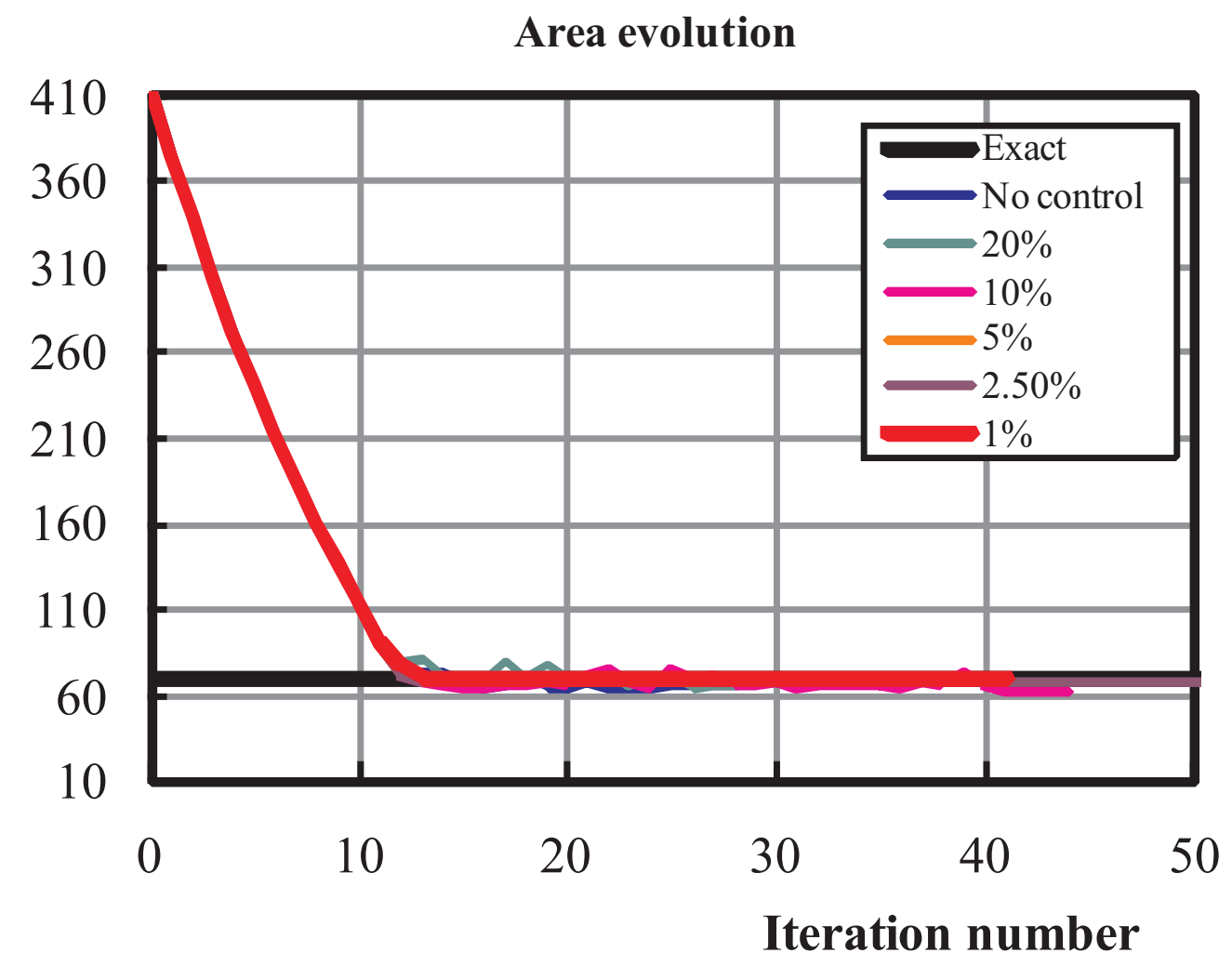

Fig. 7. Deterministic algorithm. Influence of $\gamma$ over the evolution of the objective function. 


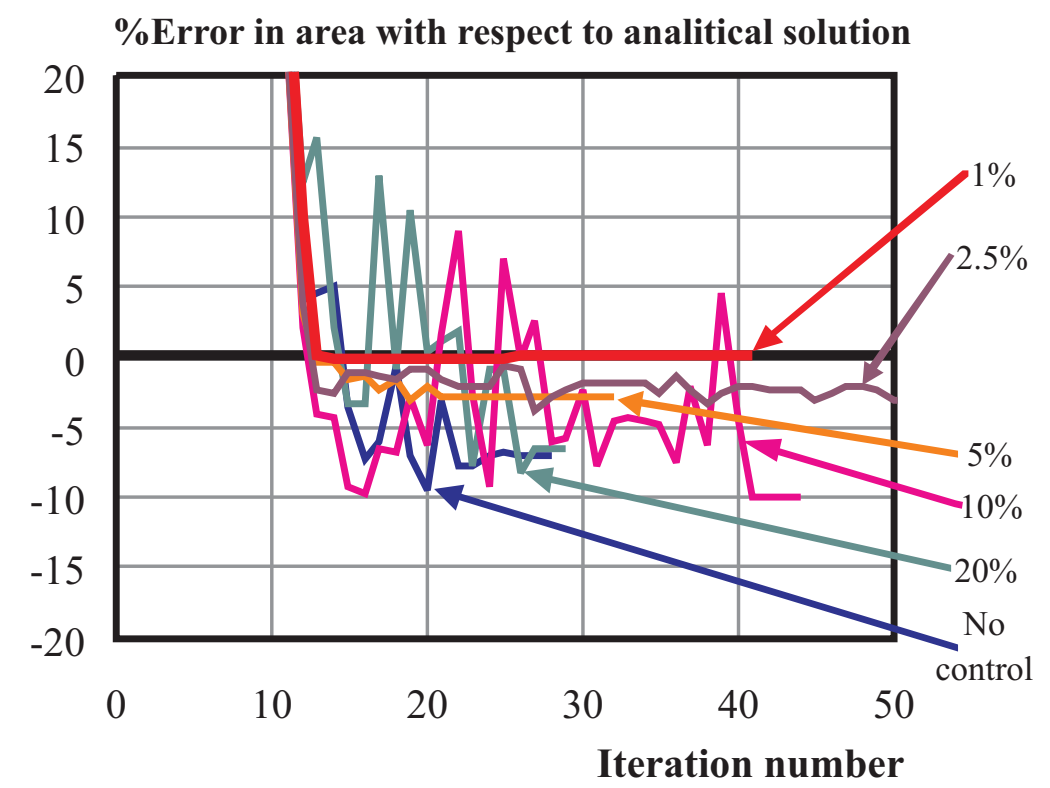

Fig. 8. Deterministic algorithm. Influence of $\gamma$ over the evolution of the difference between the obtained objective function and the analytical solution. 

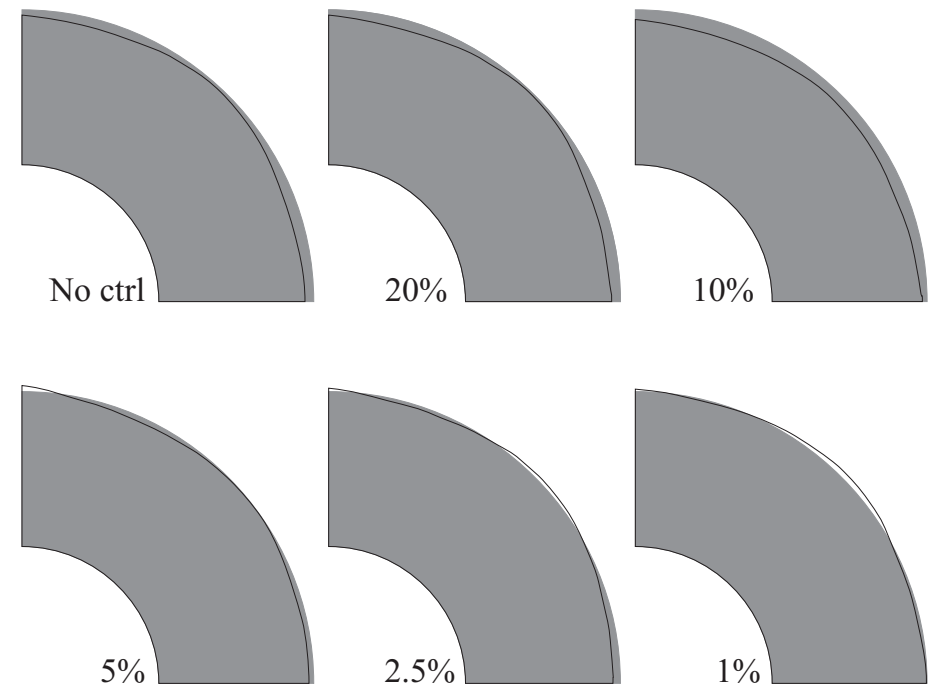

Fig. 9. Deterministic algorithm. Influence of $\gamma$ over the optimal solution found (black contour) and comparison with optimal analytical solution (shaded area). 


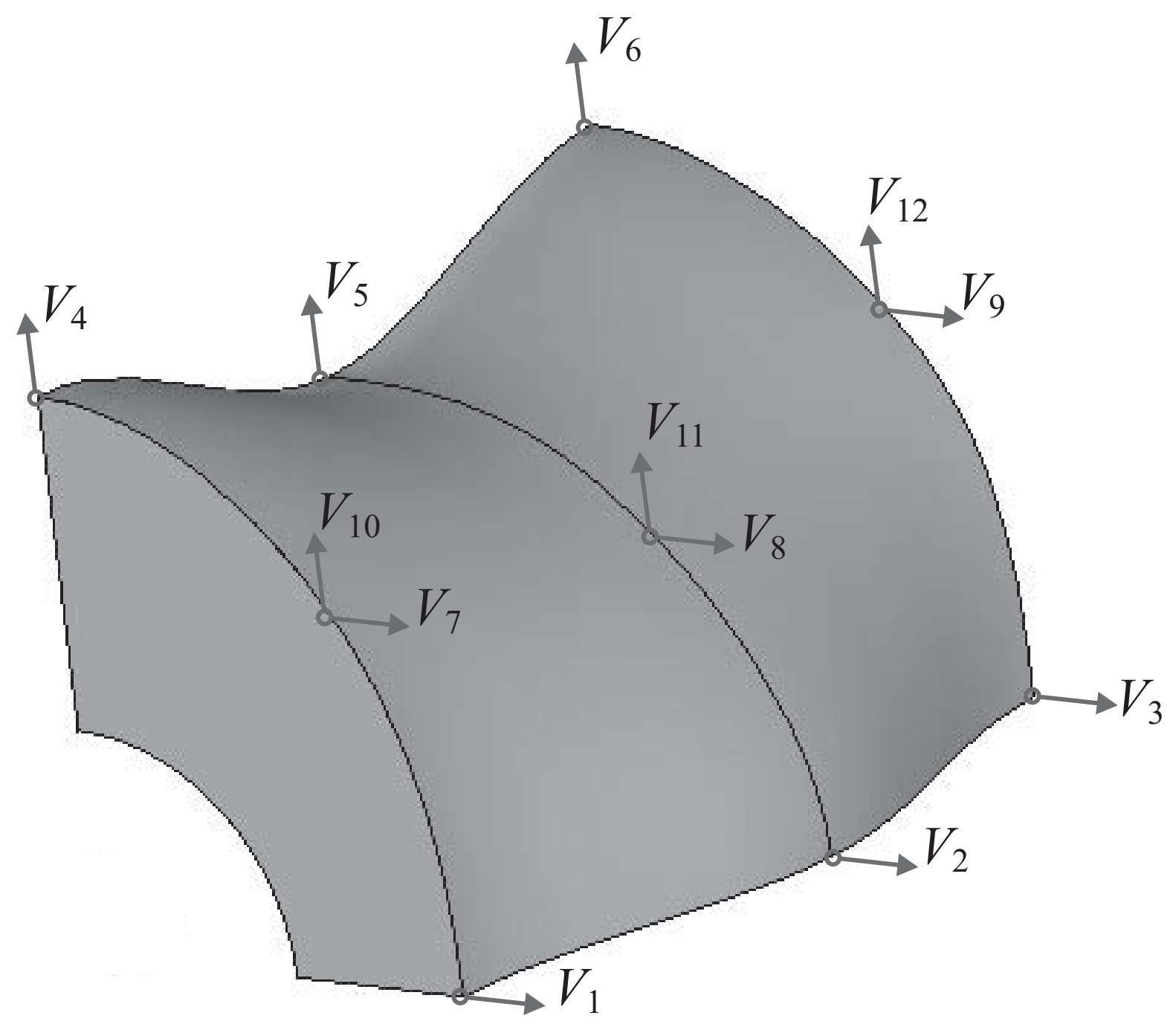

Fig. 10. 3D pipe model. 


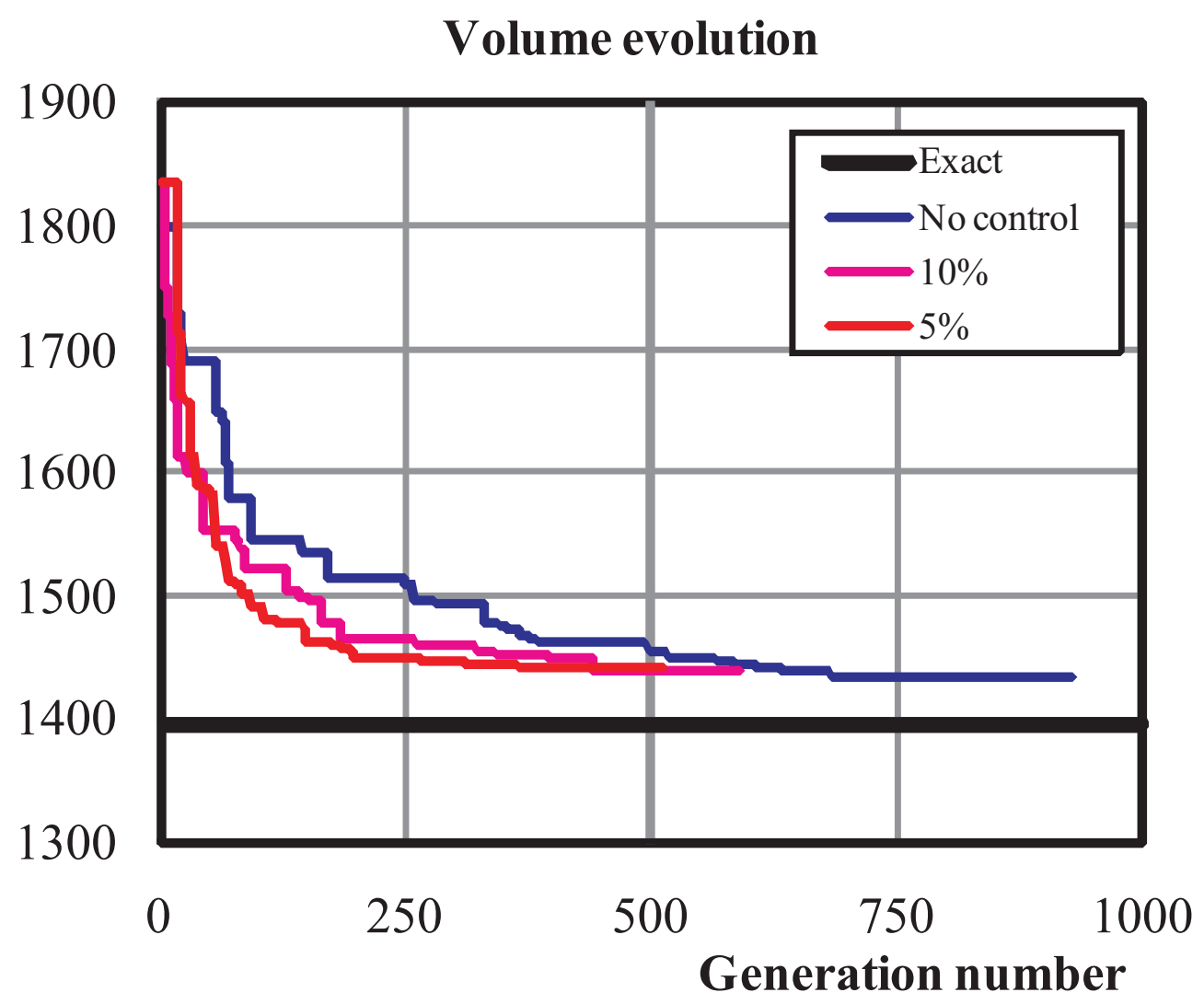

Fig. 11. 3D model. Evolutionary algorithm + Ansys ${ }^{\oplus}$. Influence of $\gamma$ over the evolution of the objective function (volume). 
$\%$ Error in volume with respect to analitical solution

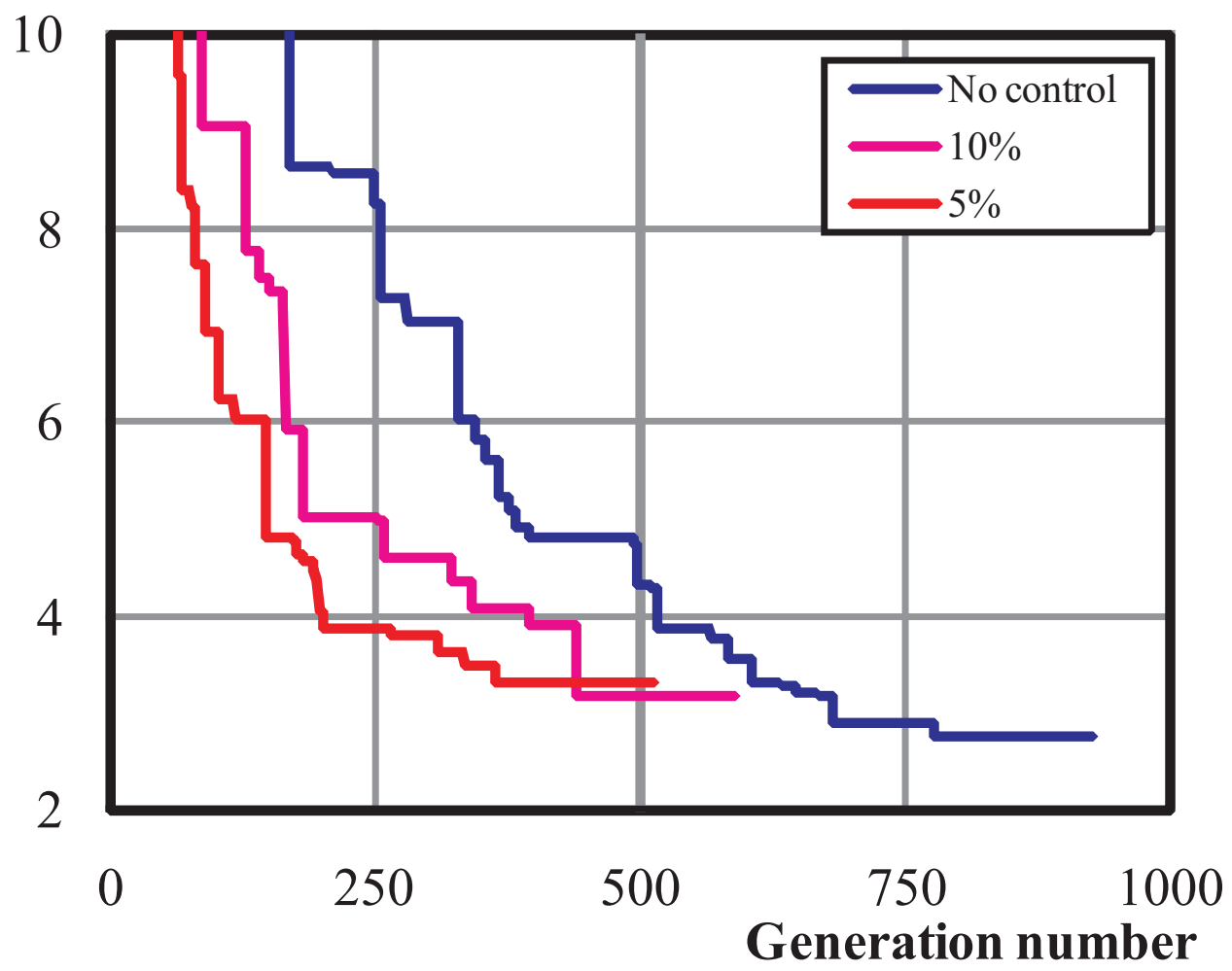

Fig. 12. 3D model. Evolutionary algorithm + Ansys ${ }^{\oplus}$. Influence of $\gamma$ over the evolution of the error in the objective function with respect to the optimal analytical solution. 

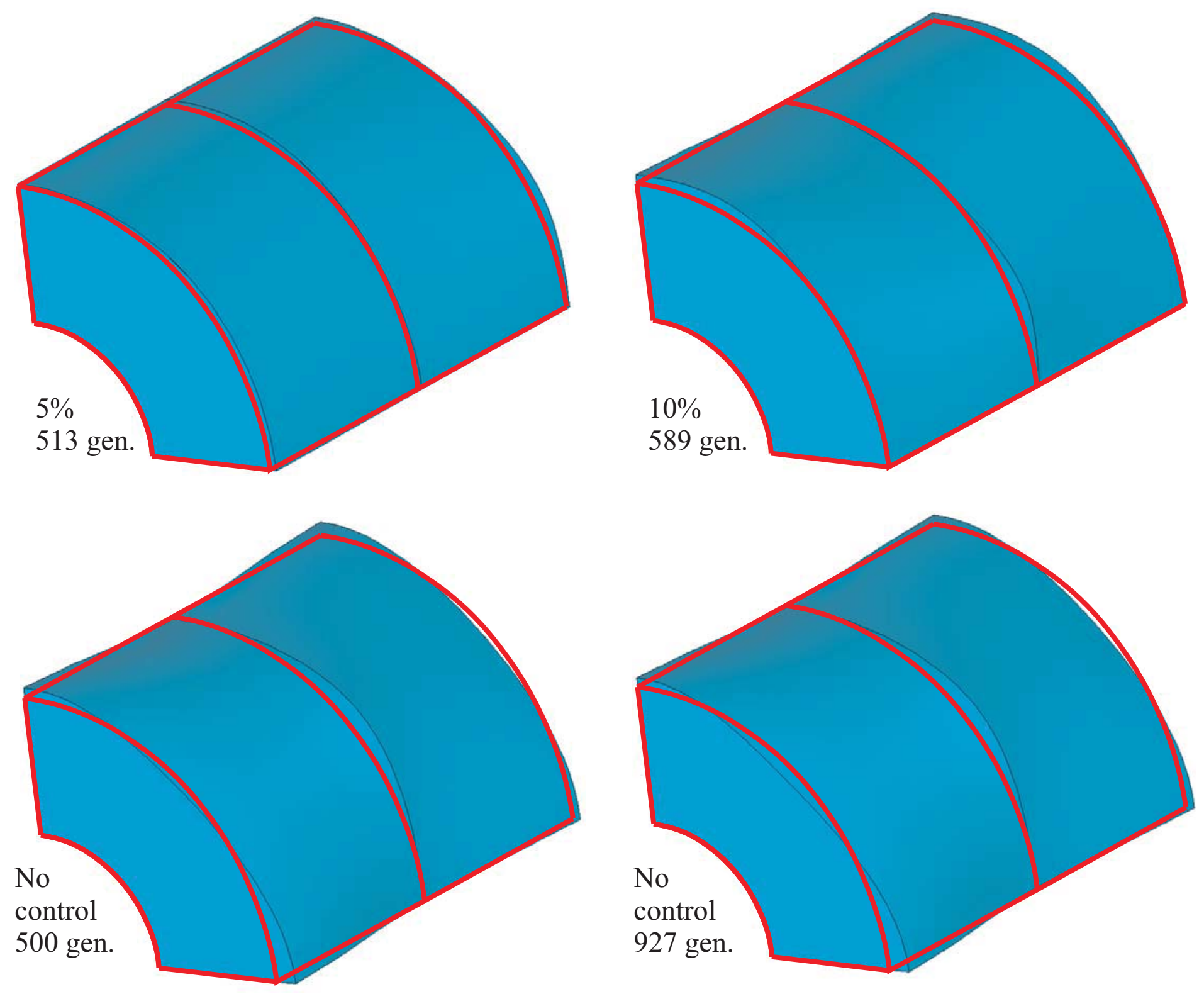

Fig. 13. 3D model. Evolutionary algorithm + Ansys ${ }^{\oplus}$. Influence of $\gamma$ over the optimal solution found and comparison with optimal analytical solution (red contour). 


\section{$\Phi \quad(\%$ Error in radius $)$}

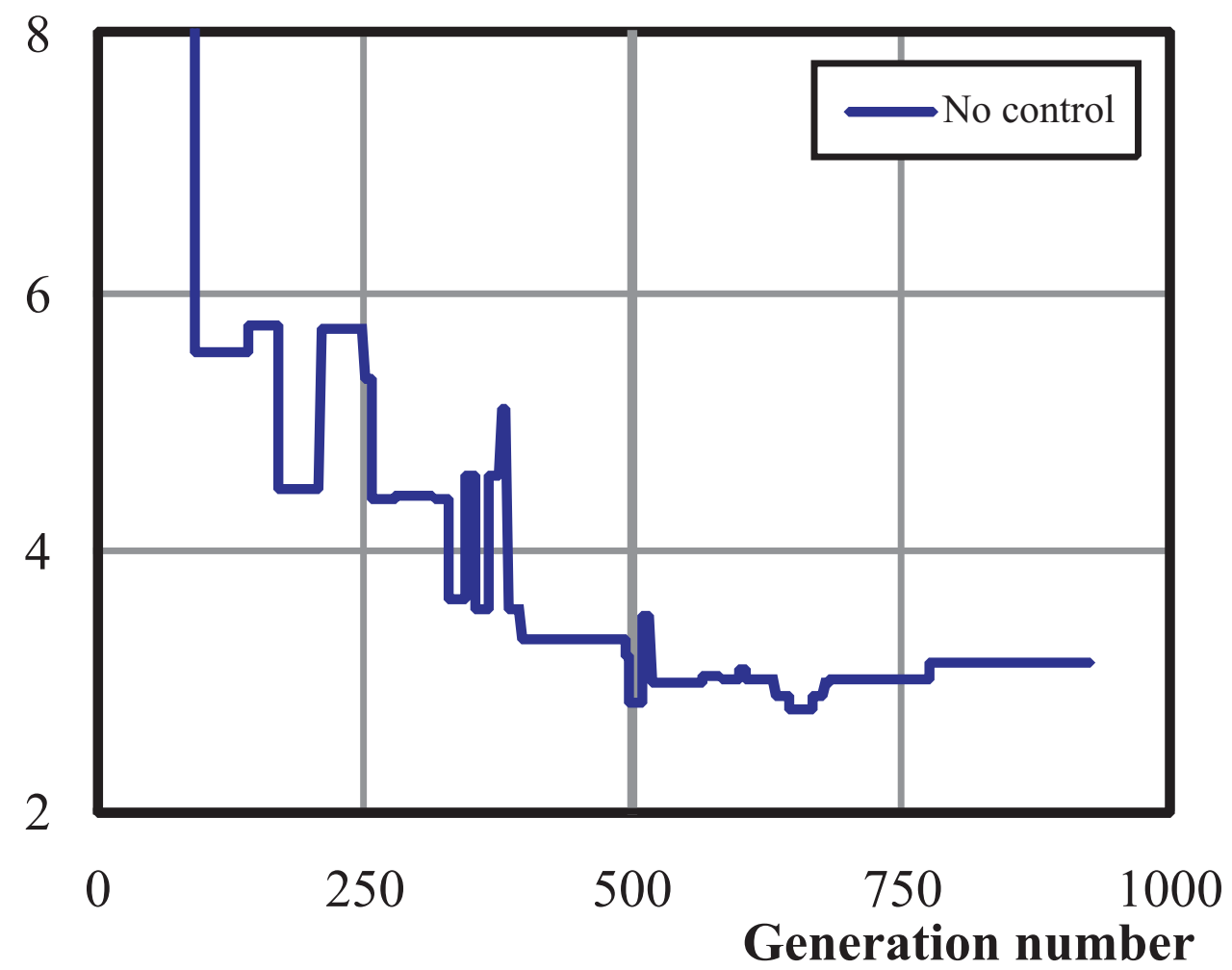

Fig. 14. 3D model. Evolutionary algorithm + Ansys ${ }^{\oplus}$. Evolution of $\Phi$ with no error control. 


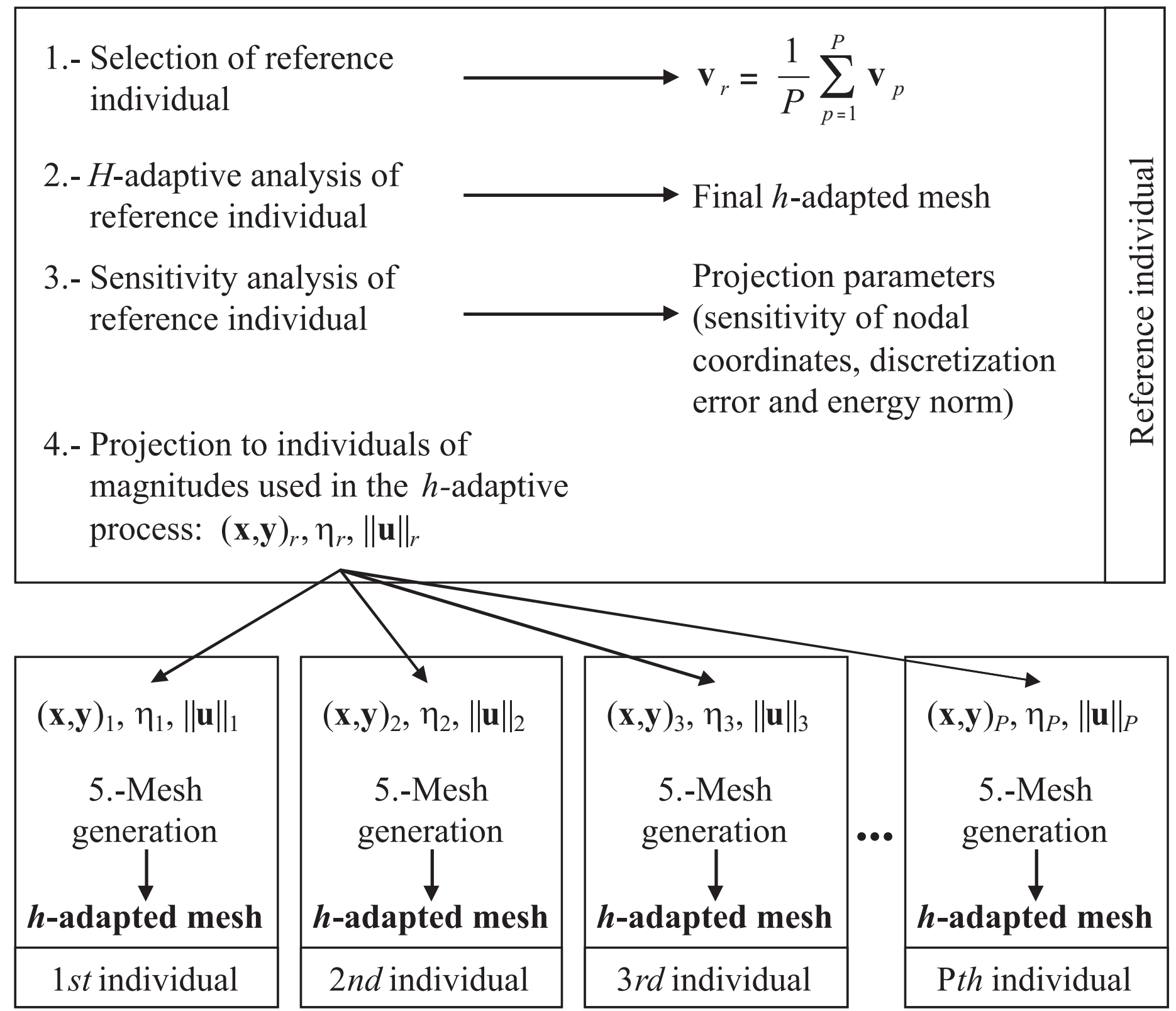

Fig. 15. Algorithm for the direct definition of h-adapted meshes for all the individuals of a generation. 


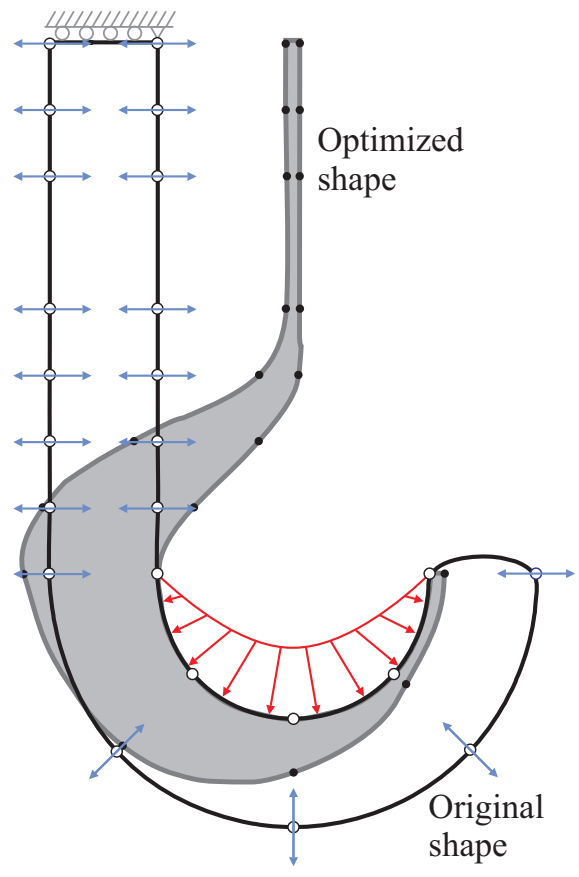

Fig. 16. Hook optimization. Original shape and design variables. Optimized shape. 


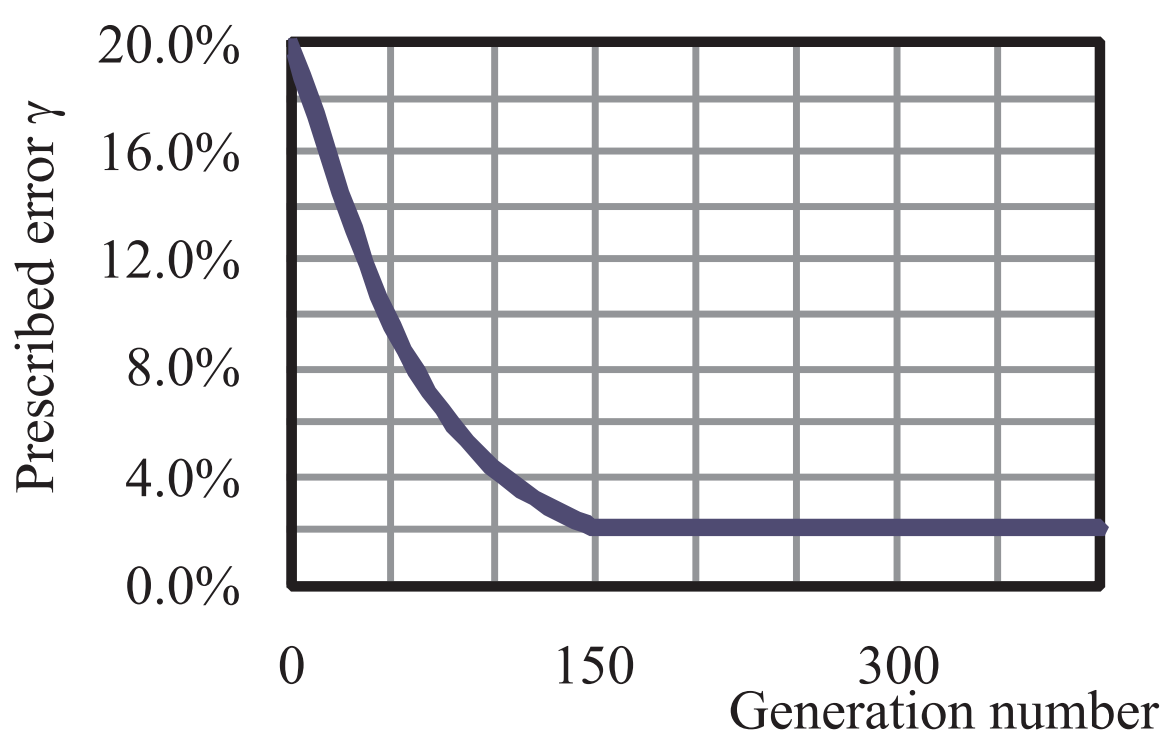

Fig. 17. Hook. Evolution of the prescribed accuracy level $\gamma$ with the progress of the optimization process. 


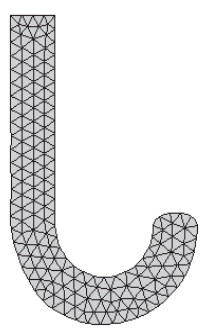

0

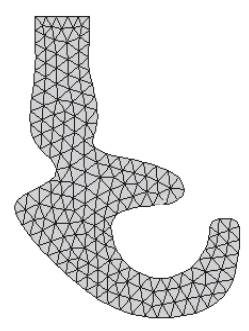

3

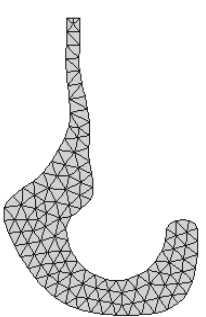

6

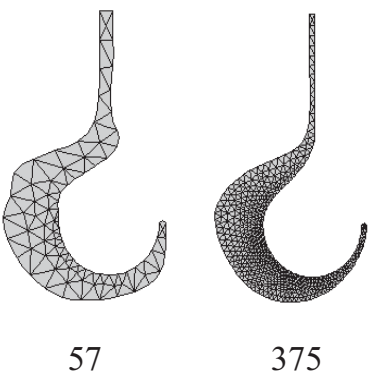

375

Fig. 18. Hook. Mesh densities used along the optimization process. The geometries represented correspond to a representative individual of a selection of generations. 

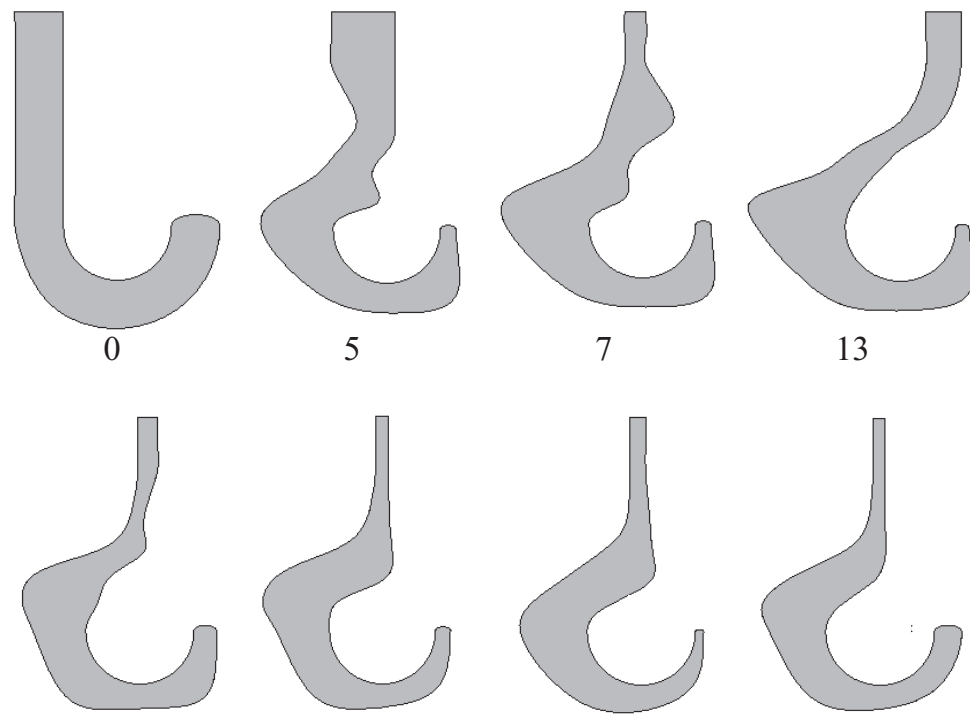

44

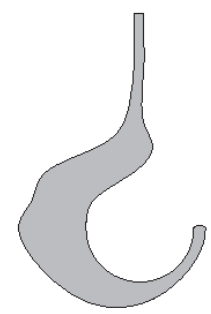

62

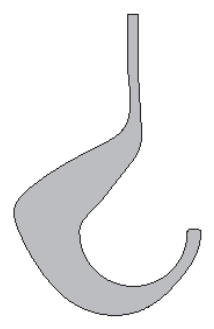

72

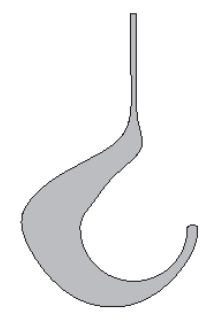

149

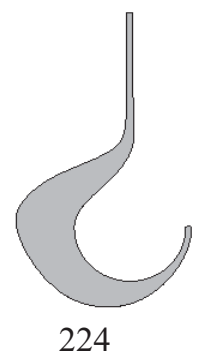

224

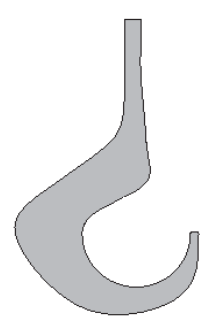

51

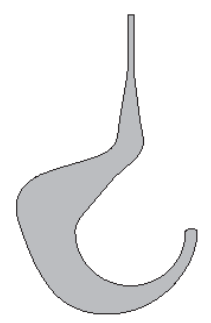

76

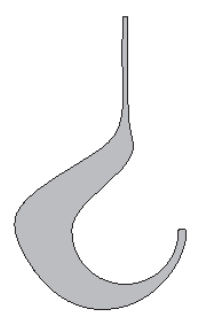

162

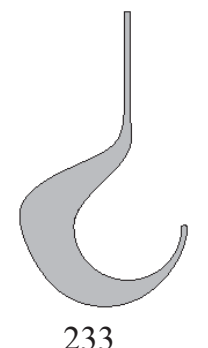

13
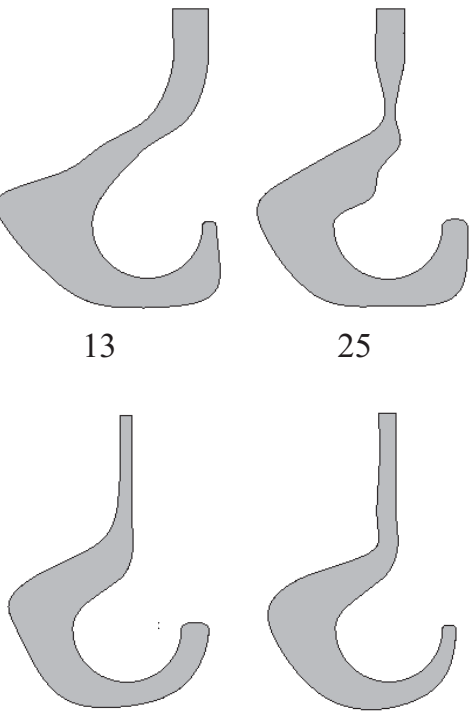

56
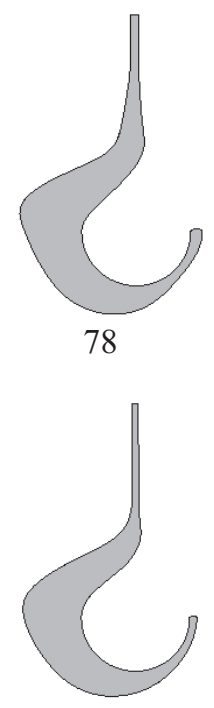

179

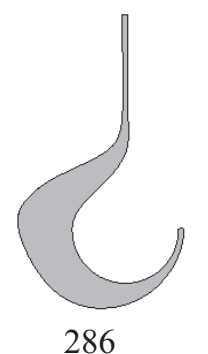

25

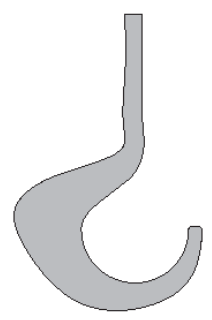

58

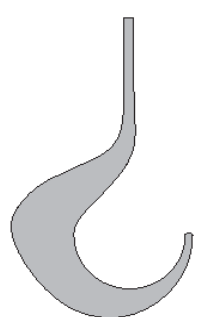

83
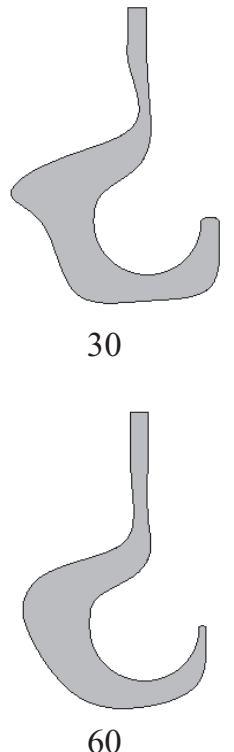

60
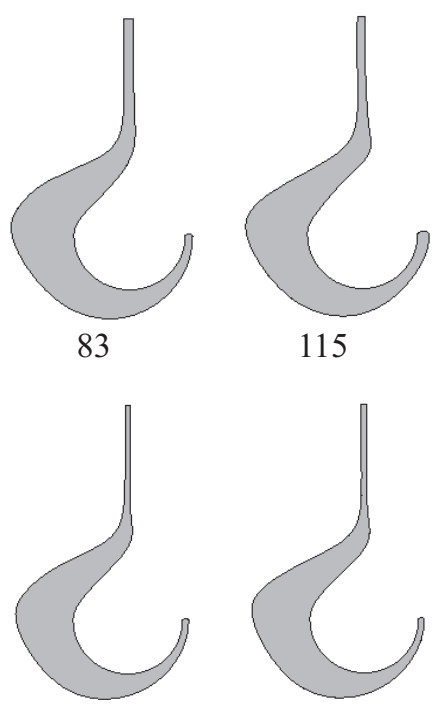

191
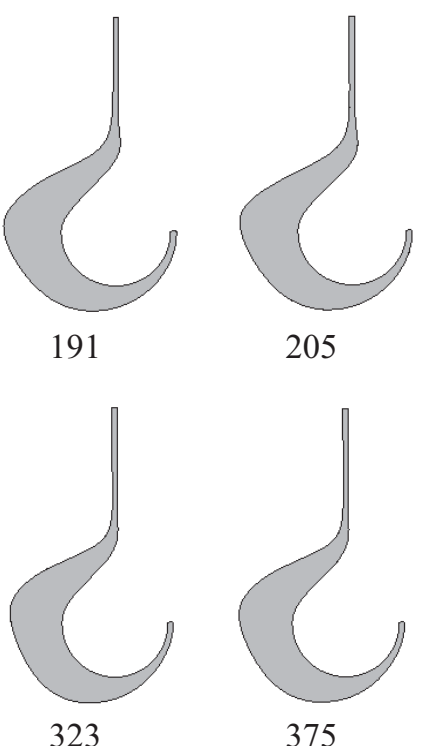

Fig. 19. Hook. Evolution of the best individual during the optimization process. The figure below each geometry indicates the generation number where the geometry was created. 


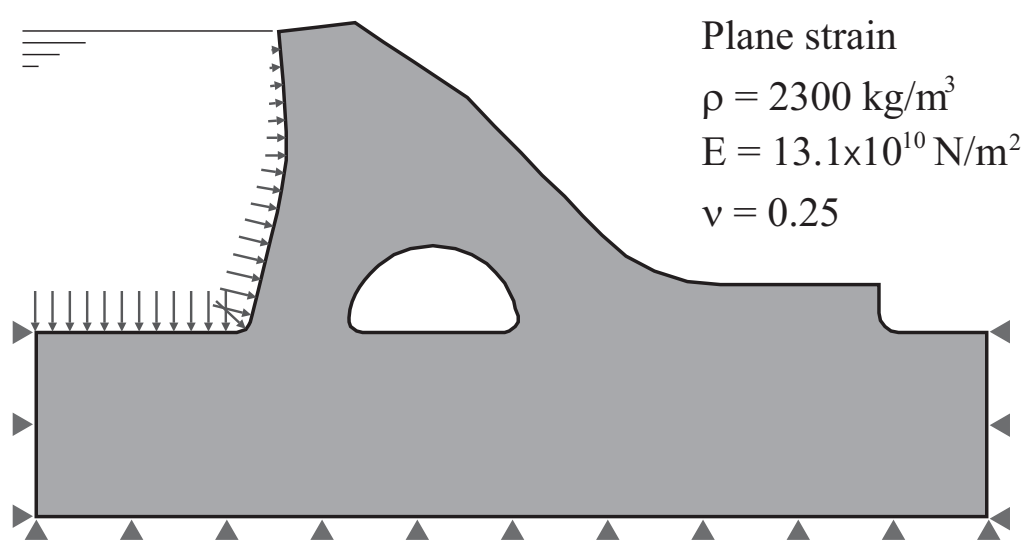

Fig. 20. Gravity dam. Analysis model. 


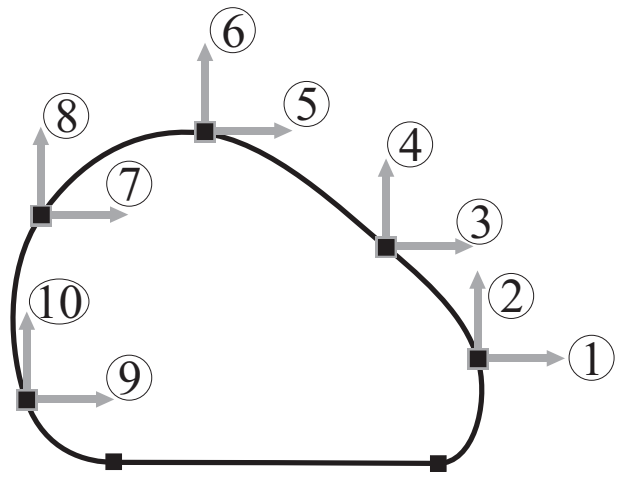

Fig. 21. Gravity dam. Design variables. 

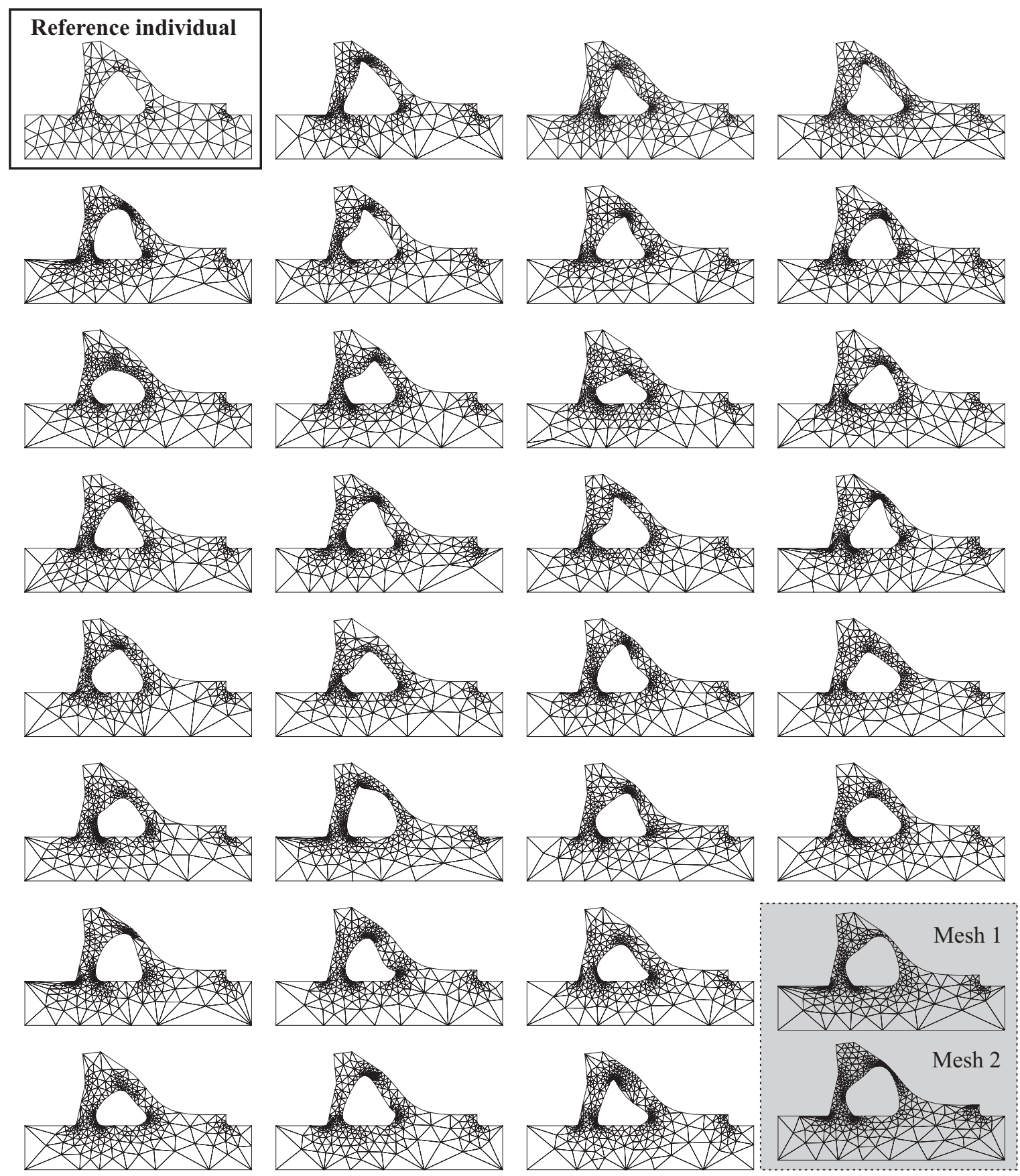

Fig. 22. Gravity dam. Generation of FE meshes for a generation of individuals by projection from a reference individual. Only the highlighted individual required remeshing. 


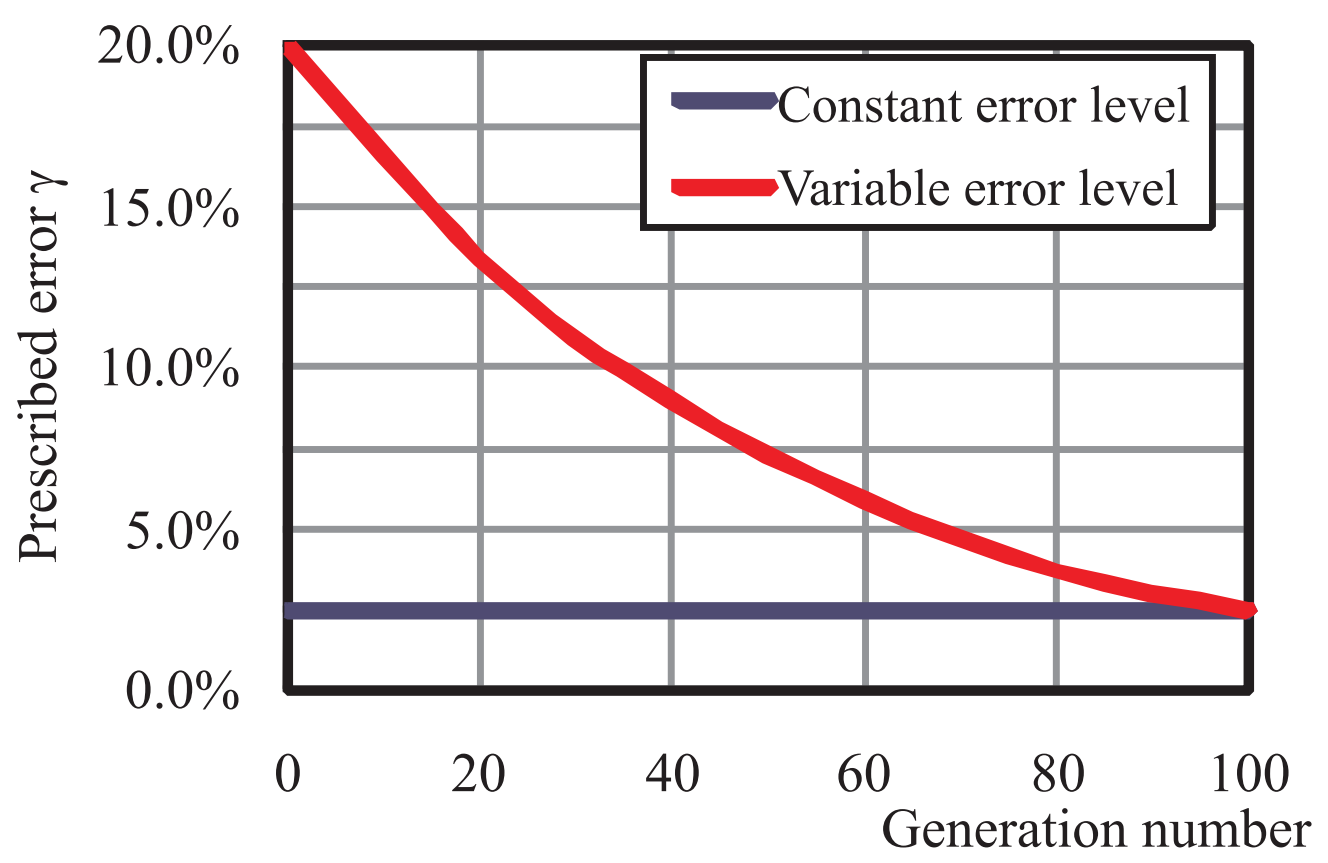

Fig. 23. Gravity dam. Evolution of the prescribed error $\gamma$. 


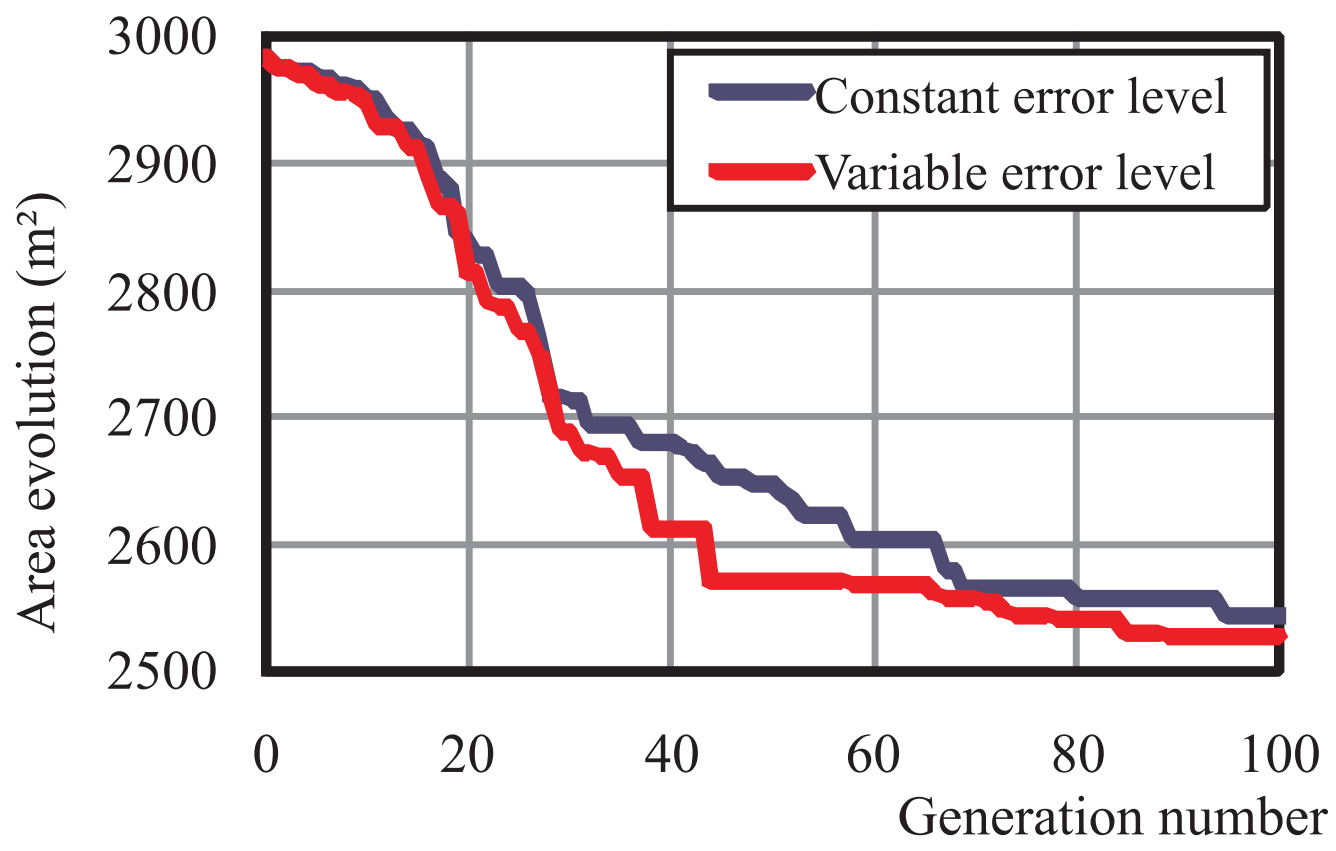

Fig. 24. Gravity dam. Area evolution. 


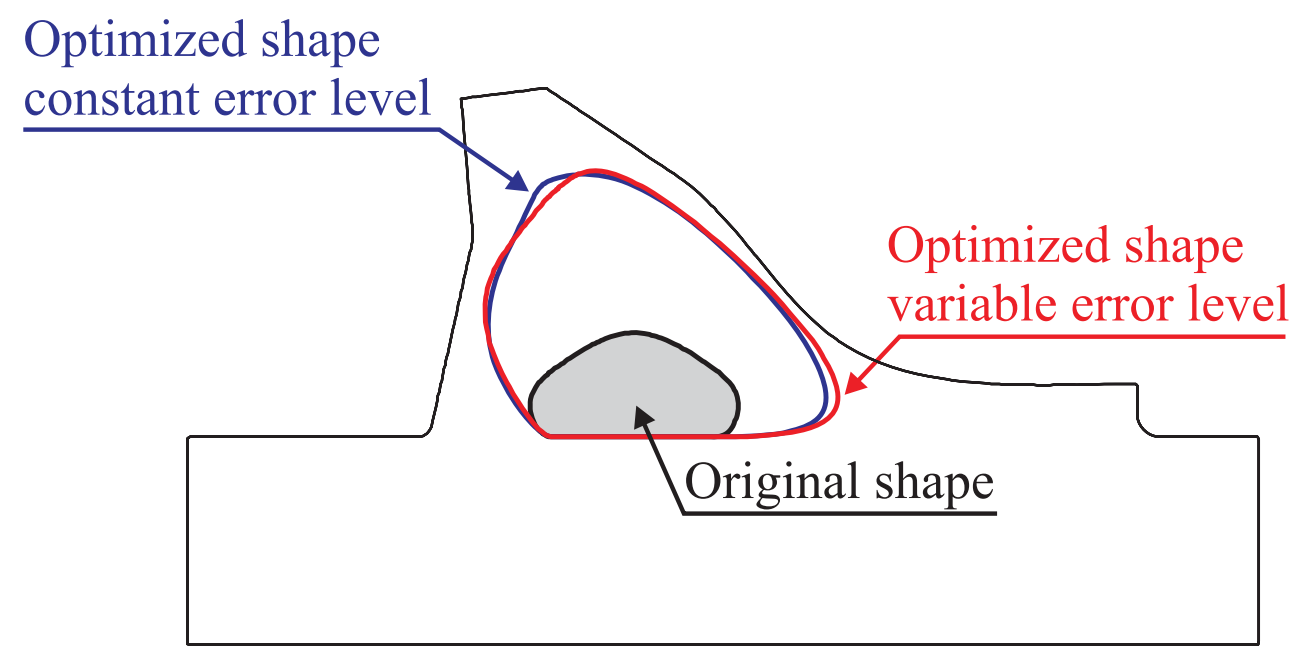

Fig. 25. Gravity dam. Optimized geometries versus original geometry. 